\title{
RIGIDITY OF GROUP ACTIONS ON HOMOGENEOUS SPACES, III
}

\author{
URI BADER, ALEX FURMAN, ALEX GORODNIK, and BARAK WEISS
}

\begin{abstract}
Consider homogeneous $G / H$ and $G / F$, for an $S$-algebraic group $G$. A lattice $\Gamma$ acts on the left strictly conservatively. The following rigidity results are obtained: morphisms, factors, and joinings defined a priori only in the measurable category are in fact algebraically constrained. Arguing in an elementary fashion, we manage to classify all the measurable $\Phi$ commuting with the $\Gamma$-action: assuming ergodicity, we find that they are algebraically defined.
\end{abstract}

\section{Introduction}

Flows on homogeneous spaces provide a rich and fruitful source of examples of dynamical systems. Most of the literature on this subject concerns actions of subgroups $H<G$ on $G / \Gamma$, where $G$ is a Lie group and $\Gamma<G$ is a lattice. In this paper we consider a situation in which the roles of the subgroups $\Gamma, H<G$ are reversed, and we study actions of a discrete subgroup $\Gamma<G$ on homogeneous spaces $G / H$. We shall mostly focus on the situation where $G$ is a Lie group or product of algebraic groups over local fields, $\Gamma<G$ is a lattice, $H<G$ is a closed (algebraic) subgroup, and $G / H$ is equipped with the Haar measure class. Our aim is to study the classification problem for such objects as:

(1) measurable $\Gamma$-equivariant maps $G / H \rightarrow G / L$,

(2) relatively probability measure-preserving (p.m.p.) $\Gamma$-quotients of $G / H$,

(3) relatively p.m.p. joinings of $\Gamma$-actions on $G / H$ with $G / L$, and

(4) quasifactors, that is, $\Gamma$-equivariant maps $G / H \rightarrow \operatorname{Prob}(G / L)$,

and seek situations where these measurable $\Gamma$-objects (maps, quotients, and joinings) are necessarily algebraic and therefore can be explicitly described. These results expand the scope of problems previously studied in this context in [28] and [7] and generalize most of the results of those papers by using new methods. Before formulating the results, we define the precise framework of this study.

DUKE MATHEMATICAL JOURNAL

Vol. 164, No. 1, ( 2015 DOI 10.1215/00127094-2860021

Received 4 August 2012. Accepted 22 April 2014.

2010 Mathematics Subject Classification. Primary 37A17; Secondary 37A35, 22E40, 22 F30. 


\section{1. $\Gamma$-spaces}

Let $G$ be a locally compact second countable group. A Borel $G$-space is a standard Borel space $(X, X)$ with a Borel action $G \curvearrowright X$, where the action map $G \times X \rightarrow X$ is Borel measurable. Two probability measures on $X$ are called equivalent if they have the same null sets. A measurable $G$-space is a Borel $G$-space $X$ equipped with a probability measure $\mu$, defined on the given Borel $\sigma$-algebra $\mathcal{X}$ on $X$, which is $G$-quasi-invariant, that is, such that $g_{*} \mu$ is equivalent to $\mu$ for every $g \in G$. We shall often write $(X,[\mu])$ to emphasize that it is only the measure class $[\mu]$ of $\mu$ that is assumed to be $G$-invariant. If $(X,[\mu])$ is a measurable $G$-space and $V$ is a Borel $G$-space, then a map $f: X \rightarrow V$ is called $G$-equivariant, or just a Borel $G$-map, if it is Borel measurable and, for every $g \in G$, for $\mu$-almost every $x \in X$,

$$
f(g \cdot x)=g \cdot f(x) .
$$

Maps $f, f^{\prime}: X \rightarrow V$ that agree $\mu$-almost everywhere will be identified, and

$$
\operatorname{Map}_{G}(X, V)
$$

denotes the set of all equivalence class of $G$-maps. In light of [33, Appendix B], any map in $\operatorname{Map}_{G}(X, V)$ is equivalent to $f: X \rightarrow V$ such that on a conull subset $X_{0} \subset X$, (1.1) holds for every $g \in G$ and every $x \in X_{0}$. If $G_{1}$ is a subgroup of $G$, then any $G$-space is automatically a $G_{1}$-space and $G$-maps are $G_{1}$-maps, yielding an injective map $\operatorname{Map}_{G}(X, Y) \rightarrow \operatorname{Map}_{G_{1}}(X, Y)$. If this map is surjective, then we write $\operatorname{Map}_{G}(X, Y)=\operatorname{Map}_{G_{1}}(X, Y)$. With the usual abuse of notation, $f \in \operatorname{Map}_{G}(X, V)$ will often mean that $f$ is an actual $G$-map, rather than an equivalence class of such maps.

A measurable $G$-space $(X,[\mu])$ is ergodic if every $G$-invariant measurable subset is $\mu$-null or $\mu$-conull. Let $T$ be a standard Borel space with the trivial $G$-action. Then $(X,[\mu])$ is ergodic if and only if every $G$-map $X \rightarrow T$ is a constant map; that is, $\operatorname{Map}_{G}(X, T) \cong T$.

\subsection{Relatively p.m.p. factors}

Let $(X,[\mu]),(Y,[\nu])$ be measurable $G$-spaces, and let $p: X \rightarrow Y$ be a $G$-map so that $\left[p_{*} \mu\right]=[\nu]$. Such a map will be called a $G$-morphism, $G$-quotient, or $G$-factor (map). We will be particularly interested in relatively measure-preserving morphisms, which we now define. Recall that if $X$ is a Borel $G$-space, then so is the space $\operatorname{Prob}(X)$ of Borel probability measures on $X$. Additionally recall that if $X, Y$ are standard Borel spaces, $\mu$ is a Borel measure on $X, p: X \rightarrow Y$ is a Borel map, and $v$ is the pushforward $p_{*} \mu$, then there is a disintegration of $\mu$, that is, a measurable map $Y \rightarrow$ $\operatorname{Prob}(X), y \mapsto \mu_{y}$ such that

$$
\mu=\int_{Y} \mu_{y} d \nu(y), \quad \text { that is, } \quad \mu(E)=\int_{Y} \mu_{y}(E) d v(y)
$$


for all measurable $E \subset X$, and such that

$$
\mu_{y}\left(p^{-1}(\{y\})\right)=1 \quad \text { for } v \text {-a.e. } y \in Y
$$

This map is unique in the sense that any two such maps differ on a null set for $v$. We now say that a $G$-morphism $p:(X,[\mu]) \rightarrow(Y,[v])$ is relatively p.m.p. if there is $\mu^{\prime} \in[\mu]$ such that, in the disintegration of $\mu^{\prime}$ with respect to $p_{*} \mu^{\prime}$, the corresponding map $Y \rightarrow \operatorname{Prob}(X)$ is $G$-equivariant, that is,

$$
\mu_{g \cdot y}=g_{*} \mu_{y} \quad(g \in G)
$$

for $\nu$-almost every $y \in Y$. If the measure class $[\mu]$ contains a $G$-invariant probability measure $\mu_{0}$, then $[v]$ contains a $G$-invariant probability measure $v_{0}=p_{*} \mu_{0}$ and $p$ is relatively p.m.p. If $G$ is a locally compact second countable group and $H<G$ is a closed subgroup, then there is a unique invariant measure class on $G / H$ induced by a Haar measure on $G$, which we will call the Haar measure class on $G / H$; the invariant measure class may or may not contain an invariant probability measure. If $L<G$ is another closed subgroup containing $H$, then the $G$-equivariant map $G / H \rightarrow G / L$, $g H \mapsto g L$ is relatively p.m.p. if and only if $L / H$ carries an $L$-invariant probability measure.

\subsection{Relatively p.m.p. joinings and quasifactors}

A relatively p.m.p. joining of two measurable $\Gamma$-spaces $\left(X_{i},\left[\mu_{i}\right]\right), i=1,2$, is a $G$-quasi-invariant probability measure $v$ on $X_{1} \times X_{2}$, so that both projections

$$
p_{i}:\left(X_{1} \times X_{2}, v\right) \rightarrow\left(X_{i}, \mu_{i}\right) \quad(i=1,2)
$$

are relatively p.m.p. maps. For instance, if $p:(X, \mu) \rightarrow(Y, v)$ is a relatively p.m.p. quotient map, then the pushforward of $\mu$ under the map $x \mapsto(x, p(x))$ gives a relatively p.m.p. joining of $(X, \mu)$ with $(Y, v)$. Another joining associated to $p:(X, \mu) \rightarrow$ $(Y, v)$ is the relatively independent self-joining of $(X, \mu)$ relative to $(Y, v)$, given by the measure

$$
\mu \times{ }_{v} \mu=\int_{Y} \mu_{y} \times \mu_{y} d v(y)
$$

on $X \times X$ (or rather on the subset $\left.X \times_{Y} X=\left\{\left(x_{1}, x_{2}\right) \in X \times X \mid p\left(x_{1}\right)=p\left(x_{2}\right)\right\}\right)$, where $\mu=\int_{Y} \mu_{y} d \nu(y)$ is the disintegration for $p: X \rightarrow Y$, that is, $\mu_{y}\left(p^{-1}(\{y\})\right)=$ 1 for $\nu$-almost every $y \in Y$.

Given measurable $G$-spaces $(X, \mu)$ and $(Y, \nu)$, we say that $(Y, \nu)$ is a quasifactor of $(X, \mu)$ if there is a $G$-map $\phi \in \operatorname{Map}_{G}(Y, \operatorname{Prob}(X)), y \mapsto \phi_{y}$ such that

$$
\mu=\int_{Y} \phi_{y} d v(y)
$$


Thus every relatively p.m.p. quotient map $p:(X, \mu) \rightarrow(Y, v)$ defines a quasifactor by disintegration.

Furthermore, any relatively p.m.p. joining $v$ of $\left(X_{1}, \mu_{1}\right)$ and $\left(X_{2}, \mu_{2}\right)$ defines a pair of quasifactor maps $X_{1} \rightarrow \operatorname{Prob}\left(X_{2}\right), X_{2} \rightarrow \operatorname{Prob}\left(X_{1}\right)$, via the disintegration of the projections $p_{i}: v \rightarrow \mu_{i}$. Hence a classification of all quasifactors may lead to a classification of relatively p.m.p. joinings and relatively p.m.p. factors. For example, if $p:(X, \mu) \rightarrow(Y, \nu)$ is a relatively p.m.p. $G$-factor and $y \mapsto \mu_{y}$ is the associated disintegration of $\mu$ with respect to $v$, then

$$
X \rightarrow \operatorname{Prob}(X), \quad x \mapsto \mu_{p(x)}
$$

is a $G$-quasifactor.

\subsection{S-algebraic groups, subgroups, actions}

Let $S$ be a finite set, for each $v \in S$ let $k_{v}$ be a nondiscrete local field of zero characteristic (i.e., $\mathbb{R}, \mathbb{C}$, or a finite extension of $\mathbb{Q}_{p}$ for some prime $p$ ), and let $\mathbf{G}_{v}$ be an algebraic group defined over $k_{v}$. Let $G=\prod_{v \in S} \mathbf{G}_{v}\left(k_{v}\right)$ be the locally compact second countable group formed by the product of the $k_{v}$-points $\mathbf{G}_{v}\left(k_{v}\right)$ of $\mathbf{G}_{v}$. We shall use the term $S$-algebraic group to describe groups $G$ that arise in this way. By an $S$-algebraic subgroup $H$ of an $S$-algebraic group $G$, we mean any locally compact subgroup $H=\prod_{v \in S} \mathbf{H}_{v}\left(k_{v}\right)$ where $\mathbf{H}_{v}<\mathbf{G}_{v}$ is a $k_{v}$-algebraic subgroup for each $v \in S$. Similarly, $V$ is an $S$-variety if $V=\prod_{v \in S} \mathbf{V}_{v}\left(k_{v}\right)$ is a product of $k_{v}$-points of $k_{v}$-varieties for $v \in S$. We shall say that $V$ is an $S$-algebraic $G$-space if $G$ is an $S$-algebraic group and $V$ is an $S$-algebraic variety equipped with a $G$-action $G \curvearrowright V$ associated to $k_{v}$-algebraic actions $\mathbf{G}_{v} \curvearrowright \mathbf{V}_{v}$ for each $v \in S$. Homogeneous $S$-algebraic spaces are $V=G / H$, where $H<G$ is an $S$-algebraic subgroup.

Any $S$-algebraic space $V$ can be considered as a Borel $G$-space, where the Borel structure of $V$ is induced by its locally compact topology. A homogeneous $S$-algebraic $G$-space, $V=G / H$, equipped with the unique $G$-invariant measure class $[\mu]_{G / H}$ is a measurable $G$-space. If $M<G$ is a closed (not necessarily $S$-algebraic) subgroup, then any $S$-algebraic space $V$ can be viewed as a Borel $M$-space and any $S$-algebraic $G$-homogeneous space $G / H$ is a measurable $M$-space.

Let $G$ be a general locally compact second countable group. A discrete subgroup $\Gamma<G$ is a lattice if $G / \Gamma$ has a $G$-invariant finite measure. One of the reasons to consider the framework of $S$-algebraic groups, rather than just algebraic groups over a single field, is that an $S$-algebraic group $G$ may contain irreducible lattices, that is, lattices that have a nondiscrete image in any proper factor of $G$. These are so-called $S$-arithmetic lattices that arise from a number field $k$, a connected semisimple $k$-group $\mathbf{G}$, and a choice of a finite set $S$ of inequivalent valuations of $k$. By letting $k(S)$ denote the ring of $S$-integers in $k$, the countable group $\Gamma=\mathbf{G}(k(S))$ 
embeds as an irreducible lattice in the $S$-algebraic group $\prod_{v \in S} \mathbf{G}\left(k_{v}\right)$. For example, $\Gamma=\mathrm{SL}_{n}(\mathbb{Z}[1 / p])$ is a lattice in the $S$-algebraic group $G=\mathrm{SL}_{n}(\mathbb{R}) \times \mathrm{SL}_{n}\left(\mathbb{Q}_{p}\right)$.

Recall that if $H, M$ are closed subgroups in some locally compact group $G$, then $M \curvearrowright G / H$ is ergodic if and only if $H \curvearrowright G / M$ is ergodic, where both spaces are considered with the Haar measure classes. If $\Gamma$ is an irreducible lattice in the product $G=\prod \mathbf{G}_{v}\left(k_{v}\right)$ formed by the $k_{v}$-points of semisimple $k_{v}$-groups and if $H=\prod \mathbf{H}_{v}\left(k_{v}\right)$ is not compact, then $H \curvearrowright G / \Gamma$ and $\Gamma \curvearrowright G / H$ are ergodic by Moore's ergodicity theorem.

\subsection{Statements of the main results}

We now present the main results for actions of a lattice $\Gamma$ in an $S$-algebraic group $G$ on $G / H$, where $H<G$ is an $S$-algebraic subgroup. We assume ergodicity of $\Gamma \curvearrowright$ $G / H$ (equivalently $H \curvearrowright G / \Gamma$ ). Let us first state the results under the following simplifying assumption:

(*) $\quad H$ has no nontrivial $S$-algebraic compact factor group.

Hereafter for any group action $G \curvearrowright S$ we denote by $S^{G}$ the set of $G$-fixed points in $S$.

THEOREM 1.1 (Equivariant Borel maps)

Let $G$ be an $S$-algebraic group, let $H<G$ be an $S$-algebraic subgroup, let $\Gamma<G$ be a lattice, and let $G / H$ be an ergodic $\Gamma$-space. Assume (*). Let $V$ be an $S$-algebraic $G$-space viewed as a Borel $\Gamma$-space. Then

$$
\operatorname{Map}_{\Gamma}(G / H, V)=\operatorname{Map}_{G}(G / H, V) \cong V^{H}
$$

where $v \in V^{H}$ corresponds to the $G$-map $f_{v}(g H)=g \cdot v$.

As an immediate corollary we obtain measure-theoretic rigidity results. Given a measurable $\Gamma$-space $(X,[\mu])$ denote by $\operatorname{Aut}(X,[\mu])$ the group of measure-class automorphisms of $X$, up to null sets, and let $\operatorname{Aut}_{\Gamma}(X,[\mu])$ denote the subgroup of $\Gamma$-equivariant ones.

We denote conjugation by $h^{g}=g^{-1} h g, H^{g}=g^{-1} H g$ and normalizers by

$$
\mathcal{N}_{G}(H)=\left\{g \in G \mid H^{g}=H\right\} .
$$

Recall that $\mathcal{N}_{G}(H) / H$ is $\operatorname{Aut}_{G}(G / H)$-the group of $G$-equivariant bijections of $G / H$ as a set. Here $n H \in \mathcal{N}_{G}(H) / H$ acts on $G / H$ by $g H \mapsto g n H$.

COROLLARY 1.2

Let $G$ be an $S$-algebraic group, let $H, H_{1}, H_{2}<G$ be $S$-algebraic subgroups satisfying (*), and let $\Gamma<G$ be a lattice whose action on $G / H_{i}$ is ergodic. Then the following statements hold. 
(a) $\operatorname{Map}_{\Gamma}\left(G / H_{1}, G / H_{2}\right)=\operatorname{Map}_{G}\left(G / H_{1}, G / H_{2}\right)$ and all such maps are given by $g H_{1} \mapsto g g_{0} H_{2}$, where $H_{1}^{g_{0}}<H_{2}$.

(b) The spaces $G / H_{1}$ and $G / H_{2}$ are isomorphic as ergodic $\Gamma$-spaces if and only if they are algebraically isomorphic; that is, $H_{1}^{g_{0}}=H_{2}$ for some $g_{0} \in G$.

(c) $\operatorname{Aut}_{\Gamma}(G / H)=\operatorname{Aut}_{G}(G / H) \cong \mathcal{N}_{G}(H) / H$.

Given an ergodic $\Gamma$-space $G / H$ as above, it is possible to classify all of its relatively p.m.p. $\Gamma$-factors.

THEOREM 1.3 (Relatively p.m.p. factors)

Let $\Gamma, H<G$ be as in Theorem 1.1, and let $p: G / H \rightarrow Y$ be a relatively p.m.p. $\Gamma$-factor. Then $(Y, v)$ is a relatively p.m.p. $G$-factor of $G / H$. More precisely, there is a closed subgroup $H \triangleleft L<G$ with $K=L / H$ compact, so that $Y \cong G / L$ and

$$
p: G / H \rightarrow Y \cong G / L \text { is given by } g H \mapsto g L .
$$

The following result shows that, under assumption $\left(^{*}\right)$, ergodic measurable $\Gamma$-spaces $G / H_{1}$ and $G / H_{2}$ have no nontrivial relatively p.m.p. joinings, unless $G / H_{1} \cong G / H_{2}$.

THEOREM 1.4 (Relatively p.m.p. joinings)

Let $\Gamma, H_{1}, H_{2}<G$ be as in Corollary 1.2. Then the ergodic $\Gamma$-spaces $G / H_{1}$ and $G / H_{2}$ admit a relatively p.m.p. joining if and only if $H_{1}$ and $H_{2}$ are conjugate in $G$.

THEOREM 1.5 (Quasifactors)

Let $\Gamma, H<G$ and $V$ be as in Theorem 1.1, and let $\phi: G / H \rightarrow \operatorname{Prob}(V)$ be a $\Gamma$-quasifactor, where $V$ is equipped with the $\Gamma$-quasi-invariant measure

$$
v=\int_{G / H} \phi_{g H} d \mu_{G / H}
$$

Then $V^{H} \neq \varnothing$ and $\phi_{g H}=g \cdot v_{0}$ for some fixed $v_{0} \in \operatorname{Prob}\left(V^{H}\right)$.

In short, the results above show that certain classes of measurable $\Gamma$-maps on a $G$-homogeneous space $G / H$ are necessarily $G$-maps, and therefore can be explicitly described using the transitivity of the $G$-action. These results depend on the assumption that the $S$-algebraic subgroup $H<G$ has no $S$-algebraic compact factors. In the case of a general $S$-algebraic subgroup $H<G$, measurable $\Gamma$-maps as above need not be $G$-maps. We shall show, however, that such maps are $M$-maps, where $M$ is some closed cocompact subgroup of $G$ containing $\Gamma$ and acting transitively on $G / H$. Moreover, $M$ is a fat complement of $H$ in $G$ as described in Definition 6.1. 
THEOREM 1.6 (Quasifactors, general case)

Let $G$ be an $S$-algebraic group, let $H<G$ be an $S$-algebraic subgroup, let $\Gamma<G$ be a lattice, and assume that $G / H$ is an ergodic $\Gamma$-space. Let $V$ be an $S$-algebraic $G$-space with a $\Gamma$-quasifactor $\phi: G / H \rightarrow \operatorname{Prob}(V)$, where $V$ is equipped with the $\Gamma$-quasi-invariant measure

$$
v=\int_{G / H} \phi_{g H} d \mu_{G / H}
$$

Then there exist a closed cocompact subgroup $M<G$, containing $\Gamma$, acting transitively on $G / H$ (and being a fat complement of $H$ as in Definition 6.1), so that $V^{M \cap H} \neq \emptyset$, and a probability measure $v_{0}$ supported on $V^{M \cap H}$ such that, for almost every $m \in M$,

$$
\phi_{m H}=m \cdot v_{0} .
$$

Moreover, if $G$ satisfies (*), then $M$ has finite index in $G$.

When $M$ acts transitively on $G / H$, for any $g \in G$ there is $m \in M$ such that $g H=m H$, and any two such elements of $M$ differ by right-multiplication by an element of $M \cap H$. Therefore for $v \in V^{M \cap H}$, the map $f_{v}(g H)=m \cdot v$ is well defined and is an $M$-map. Clearly all $M$-maps $G / H \rightarrow V$ arise in this way.

COROLLARY 1.7 (Equivariant maps, general case)

Let $\Gamma, H<G$, and $V$ be as above. Then there exists $M<G$ as in Theorem 1.6, so that

$$
\operatorname{Map}_{\Gamma}(G / H, V)=\operatorname{Map}_{M}(G / H, V) \cong V^{M \cap H}
$$

COROLLARY 1.8 (Relatively p.m.p. factors, general case)

Let $\Gamma, H<G$ be as above, and let $p: G / H \rightarrow Y$ be a relatively p.m.p. $\Gamma$-factor. Then there exist $M<G$ as in Theorem 1.6 and a closed subgroup $M \cap H \triangleleft L<M$ so that $L /(M \cap H)$ has a finite $L$-invariant measure, $Y \cong M / L$, and

$$
p: G / H \rightarrow Y \cong M / L \text { is given by } m H \mapsto m L \quad(m \in M) .
$$

\subsection{Previous results}

The general inspiration for the questions considered here is the pioneering work of Marina Ratner [20]-[22] where questions of measurable isomorphism, classification of factors, and joinings were studied for actions of unipotent subgroup $H<G$ on $G / \Gamma$, where $\Gamma$ is a lattice in a Lie group $G$. (These and more general results can also be deduced from the general Ratner's theorem [23] (see also [31]).) The assumption 
that $H<G$ is unipotent is very important for these results, as they fail for the diagonal subgroup $A<\mathrm{SL}_{2}(\mathbb{R})$. It should also be emphasized that these are p.m.p. actions.

Questions of measure-theoretic rigidity for the $\Gamma$-action on infinite measure homogeneous spaces $G / H$ were addressed by Shalom and Steger [28]. In this (unpublished) work, the authors obtained a number of rigidity results by using representationtheoretic techniques, including the rigidity of lattices in $\mathrm{SL}_{2}(\mathbb{R})$ acting on $\mathbb{R}^{2}$ and the classification of relatively p.m.p. $\Gamma$-factors of $\mathbb{R}^{n}$, where $\Gamma<\mathrm{SL}_{n}(\mathbb{R})$ is a lattice.

In [7] further results were obtained for $\Gamma$-actions on $G / H$ by using purely dynamical methods (alignment property). These results included classification of centralizers, relatively p.m.p. quotients, and joinings for a particular type of homogeneous space $G / H$. In particular, in [7] the group $G$ was assumed to be semisimple, $H<G$ to be "super-spherical," and $G / H$ to carry an infinite $G$-invariant measure.

The present paper provides a more systematic study of $\Gamma$-actions on $G / H$, reproving most of the results of [7] and [28] and significantly expanding the scope of the spaces $G / H$ and of the questions. In particular, we impose no restrictions on $G$ and $H$ except for being $S$-algebraic. For instance, even the very special case with $G=$ $\mathrm{SL}_{2}(\mathbb{R})$ and $H_{1}=H_{2}=A$ (the group of diagonal matrices) of Corollary 1.2 is new. The new questions include equivariant maps to $S$-algebraic $G$-spaces (Theorems 1.1 and Corollary 1.7) and classification of quasifactors (Theorems 1.5 and 1.6).

Our results bear some similarity to Margulis's factor theorem [13] that asserts that all measurable $\Gamma$-equivariant quotients of $G / P$ are $G$-equivariant and therefore have the form $G / P \rightarrow G / Q, g P \mapsto g Q$, where $P<Q<G$ are parabolic subgroups. However, the similarity is only superficial, as the context, phenomenology, and idea of the proof are completely different. Margulis's factor theorem concerns a higher rank semisimple group $G$ and a parabolic subgroup $P<G$, but the quotient maps are not assumed to be (and never are) relatively p.m.p. This is similar to related work on factor theorems by Zimmer [32], Nevo and Zimmer [16], and Bader and Shalom [2].

\subsection{Some ideas in the proofs}

If $H$ is a subgroup of $G$, then one may restrict any $G$-space to obtain an $H$-space, and there is a complementary operation of inducing an $H$-space to obtain a $G$-space. These operations have been extensively studied in other contexts in representation theory and ergodic theory. In Section 2 we adapt them to our framework and establish an analogue of Frobenius reciprocity, which allows us to convert questions about $\Gamma$ maps, where $\Gamma$ is a lattice, to questions about $H$-maps, where $H$ is algebraic. Then, to study algebraic actions, we apply generalizations of the classical Borel density theorem [4], which says that if $G$ is a semisimple $S$-algebraic group with no compact factors and $\Gamma<G$ is a lattice, then $\Gamma$ is Zariski-dense in $G$. This theorem was generalized by a number of authors (see [6], [8], [27], [29]), and it was realized that this 
phenomenon is related to the classification of $G$-invariant probability measures on $V$, where $V$ is an $S$-algebraic $G$-space, leading to a more abstract version which says that every such measure must be supported on the set of fixed points, that is,

$$
\operatorname{Prob}(V)^{G}=\operatorname{Prob}\left(V^{G}\right) \text {. }
$$

We obtain one such generalization (Theorem 3.1) as a straightforward corollary of previous work. Frobenius reciprocity and Borel density already suffice to imply our results when $H$ satisfies (*). To obtain our results in the general case, we need a more general version of Borel density (Theorem 6.2), which we call relative Borel density. Theorem 6.2 is the main technical innovation in our paper. Its proof relies on classical arguments, along with a detailed study of the Mautner envelope and a general version of ergodic decomposition.

\subsection{Some additional results}

Our discussion so far has focused on actions of lattices $\Gamma<G$ on homogeneous spaces $G / H$, where $H$ is an $S$-algebraic subgroup of an $S$-algebraic group $G$. In fact, we have only used the fact that $G / \Gamma$ carries a finite $G$-invariant measure, and the results apply verbatim to actions of not necessarily discrete closed subgroups $L$ of $G$ provided $G / L$ carries a finite $G$-invariant measure. Such subgroups $L<G$ are said to be of finite covolume in $G$.

However, the finite covolume assumption is not necessary for the results as above to hold; it is primarily used in arguments involving Borel's density theorem, where the existence of finite invariant measure provides recurrence via the Poincaré recurrence theorem. In some situations one can exploit recurrence phenomena for actions of subgroups $\Gamma<G$ of infinite covolume on some $G / H$ to prove results analogous to [7, Theorem B].

A family of such examples appears in rank-one real Lie groups $G$; namely, $G=$ $\operatorname{Isom}_{+}\left(\mathbf{H}_{k}^{n}\right)$ - the group of orientation-preserving isometries of a symmetric space of rank-one $\mathbf{H}_{k}^{n}$ with $k$ denoting $\mathbf{R}, \mathbb{C}$, quaternions $\mathbb{H}$, or octonions $\mathbf{O}$ with $n=2$. We denote by $A<G$ the one-parameter Cartan subgroup and by $K$ a maximal compact subgroup, so that $\mathbf{H}_{k}^{n} \cong G / K$, and we denote by $C=Z_{K}(A)$ the centralizer of $A$ in $K$. Then $C A=Z_{G}(A)$ is the centralizer of $A$ in $G$. The $G$-space $G / Z_{G}(A)$ is the space of oriented geodesic lines in $\mathbf{H}_{k}^{n}$; it can also be identified with the space of pairs of distinct points

$$
G / \mathcal{Z}_{G}(A) \cong \partial^{(2)} \mathbf{H}_{k}^{n}=\partial \mathbf{H}_{k}^{n} \times \partial \mathbf{H}_{k}^{n} \backslash \Delta
$$

representing limits geo $( \pm \infty)=\lim _{t \rightarrow \pm \infty} \operatorname{geo}(t)$ of a geodesic geo(-) in $\mathbf{H}_{k}^{n}$.

Let $\Gamma$ be a torsion-free discrete subgroup in $G$. Then the right $A$-action on $\Gamma \backslash G / C$ (resp., on $G / A$ ) corresponds to the geodesic flow on the unit tangent bundle (resp., frame bundle) to the $k$-hyperbolic manifold $\Gamma \backslash G / K=\Gamma \backslash \mathbf{H}_{k}^{n}$. Recall 
that the action $A \curvearrowright \Gamma \backslash G / C$ (resp., $A \curvearrowright \Gamma \backslash G$ ) is ergodic if and only if the action $\Gamma \curvearrowright G / Z_{G}(A)$ (resp., $\Gamma \curvearrowright G / A$ ) is ergodic.

If $\Gamma$ is a lattice in $G$, that is, if $\operatorname{vol}\left(\Gamma \backslash \mathbf{H}_{k}^{n}\right)<+\infty$, then the above actions are ergodic, and one obtains

$$
\begin{aligned}
\operatorname{Aut}_{\Gamma}(G / A) & =\operatorname{Aut}_{G}(G / A)=\mathcal{N}_{G}(A) / A, \\
\operatorname{Aut}_{\Gamma}\left(G / Z_{G}(A)\right) & =\operatorname{Aut}_{G}\left(G / \mathcal{Z}_{G}(A)\right)=\mathcal{N}_{G}(A) / \mathcal{Z}_{G}(A)=\{1, F\},
\end{aligned}
$$

where $F$ is the involution corresponding to the "time reversal" of the geodesic flow. For the case of $G / A$ this follows from Corollary 1.2(c) because $A$ has (*), and the case of $G / C A$ follows from Theorem 1.7 because $G$ has (*) and has nonproper subgroups of finite index.

We claim that the same conclusions hold for infinite covolume discrete subgroups $\Gamma<G$ provided the actions are ergodic. Examples with this behavior exist in the real and complex hyperbolic spaces, namely, for $G=\operatorname{Isom}_{+}\left(\mathbf{H}_{\mathbf{R}}^{n}\right) \simeq \operatorname{SO}(n, 1)$ and Isom $_{+}\left(\mathbf{H}_{\mathbf{C}}^{n}\right) \simeq \mathrm{SU}(n, 1)$. These groups $G$ contain many lattices $\Lambda<G$ with infinite abelianization (cf. [11], [1]), including cocompact torsion-free lattices. If $\Lambda$ is such a lattice and $\Gamma=\operatorname{Ker}\left(\Lambda \rightarrow \mathbf{Z}^{d}\right)$ with $d=1,2$, then the geodesic flow on the infinite volume manifold $\Gamma \backslash \mathbf{H}_{k}^{n}$ is ergodic (see [25], [10]). This gives examples of infinite covolume discrete subgroups $\Gamma<G$ for which the action

$$
\Gamma \curvearrowright G / C A \cong \partial^{(2)} \mathbf{H}_{k}^{n}
$$

is ergodic and, in particular, conservative.

THEOREM 1.9

Let $G=\mathrm{SO}(n, 1)$ or $\mathrm{SU}(n, 1)$, and let $\Gamma<G$ be a discrete subgroup with an ergodic action on $G / A$. Then

$$
\operatorname{Aut}_{\Gamma}(G / A)=\operatorname{Aut}_{G}(G / A)=\mathcal{N}_{G}(A) / A
$$

and

$$
\operatorname{Aut}_{\Gamma}\left(G / \mathcal{Z}_{G}(A)\right)=\operatorname{Aut}_{G}\left(G / \mathcal{Z}_{G}(A)\right)=\mathcal{N}_{G}(A) / \mathcal{Z}_{G}(A)=\{\operatorname{Id}, F\},
$$

where $F$ denotes the flip $F:(x, y) \mapsto(y, x)$ on $G / Z_{G}(A) \cong \partial^{(2)} \mathbf{H}_{k}^{n}$.

\section{Remark 1.10}

The above result is also valid for the remaining rank-one groups $\operatorname{Sp}(n, 1) \simeq \operatorname{Isom}\left(\mathbf{H}_{\mathbb{H}}^{n}\right)$ and $\mathrm{F}_{4(-20)} \simeq \operatorname{Isom}\left(\mathbf{H}_{\mathbb{O}}^{2}\right)$, but for these groups $G$, the ergodicity condition forces the discrete subgroup $\Gamma<G$ to be a lattice; hence, these cases are already covered by Theorem 1.1. Let us sketch the argument showing that, for $G \simeq \operatorname{Sp}(n, 1)$ and $\mathrm{F}_{4(-20)}$ 
for any discrete $\Gamma<G$ with $\operatorname{vol}(G / \Gamma)=+\infty$ the $A$-flow on $G / \Gamma$, the imposed ergodicity assumption as in Theorem 1.9 fails. To this end choose a $K$-invariant positive function $f \in L^{2}(G / \Gamma)$ with $f(x) \rightarrow 0$ if and only if $x \rightarrow+\infty$ in $G / \Gamma$. Since the groups $G$ in question have Kazhdan's property (T) and $L^{2}(G / \Gamma)$ has no $G$-invariant vectors, for any fixed $e \neq a \in A$ there is $c>0$ so that

$$
\int_{G / \Gamma} f\left(a^{n} \cdot x\right) f(x) d m(x)<e^{-c n} \quad(n \geq 1) .
$$

It follows that, for any $c^{\prime}<c$, one has

$$
\int_{G / \Gamma}\left(\sum_{n=1}^{\infty} e^{c^{\prime} n} f\left(a^{n} \cdot x\right) f(x)\right) d m(x)<+\infty,
$$

and therefore, for almost every $x \in G / \Gamma$ one has $f\left(a^{n} . x\right)<e^{-c^{\prime} n}$ for $n \geq n\left(x, c^{\prime}\right)$. In particular, $a^{n} . x \rightarrow \infty$ in $G / \Gamma$. Consequently the $A$-actions on $G / \Gamma$ and on $C \backslash G / \Gamma$ are dissipative.

Another possible modification of our basic setup concerns the assumptions on $H<G$, namely, instead of assuming $H$ to be an $S$-algebraic subgroup, one might consider a general closed subgroup, or a discrete subgroup as an extreme case. More specifically, consider the space of $\Gamma$-equivariant measurable maps

$$
\operatorname{Map}_{\Gamma}(G / \Lambda, G / \Delta)
$$

where $\Lambda, \Delta<G$ are discrete subgroups and $\Gamma$ is a lattice in $G$. As before, the source space $G / \Lambda$ is equipped with the Haar measure class and is viewed as an ergodic $\Gamma$-space, whereas the target space $G / \Delta$ may be viewed either as a measurable $\Gamma$-space or as a Borel $\Gamma$-space.

\section{THEOREM 1.11}

Let $G$ be a connected noncompact simple real Lie group, let $\Gamma<G$ be a lattice, and let $\Lambda, \Delta<G$ be discrete subgroups, with $\Lambda$ Zariski-dense in $G$. In addition assume one of the following:

(RS) $\Lambda$ is a lattice in a subgroup of $G$ generated by unipotent elements, or

(BQ) $\Delta$ is a contained in a lattice $\Delta_{0}<G$.

Then any element of $\operatorname{Map}_{\Gamma}(G / \Lambda, G / \Delta)$ is either:

(a) $\quad a G$-map $g \Lambda \mapsto g a \Delta$, where $a \in G$ is such that $a^{-1} \Lambda a<\Delta$, or

(b) a constant map $g \Lambda \mapsto a \Delta$, where $a \in G$ is such that $a^{-1} \Gamma a<\Delta$. 


\subsection{Positive characteristic}

It would be interesting to know the extent to which our assumption that $k$ is of characteristic zero is essential. Our basic results proved under condition (*) (i.e., Theorems $1.1,1.3,1.4$, and 1.5 and Corollary 1.2) follow by our arguments, combined with a version of Borel's density theorem proved by Shalom [27]. In the proof of our more general results we used the characteristic zero assumption several times (particularly in Section 5), and it would be interesting to find the precise conditions under which the results extend to arbitrary characteristic.

\subsection{Organization of the paper}

In Section 2 we introduce the category of measurable $G$-spaces and establish a version of Frobenius reciprocity that will play a crucial role in the proof. Then in Section 3 we discuss the Borel density theorem and, in Section 4, give a self-contained proof of our main results under the assumption that $H$ has no compact algebraic quotients. Sections 2-4 exhibit our main ideas and quickly prove a substantial part of our results, avoiding technicalities.

The proof in full generality requires further preparation. In Section 5 we introduce the notions of the Mautner envelope and establish a general version of the Mautner property (Theorem 5.6), which is of independent interest. In Section 6 we discuss fat complements and deduce the relative Borel density theorem. The proofs of the remaining results are given in Section 7. As we show, they follow easily from the relative Borel density theorem. In Section 8, we give several examples to demonstrate that the assumptions in the main theorems are essential. Finally, in the Appendix, we summarize well-known results on the space of ergodic components and deduce some corollaries.

\section{Frobenius reciprocity}

Let $G$ be a locally compact second countable group. Denote by $\mathscr{B}_{G}$ the category of all Borel $G$-spaces. The objects of this category are Borel actions on standard Borel spaces $G \curvearrowright X$, and morphisms are Borel $G$-maps.

We shall denote by $\mathcal{M} \mathcal{P} \mathcal{P}_{G}$ the category of measurable $G$-spaces. The objects of this category are measure class-preserving actions of $G$ on standard probability spaces $G \curvearrowright(X,[\mu])$, and the morphisms are $G$-morphisms $p: X \rightarrow Y$ for which $[v]=\left[p_{*} \mu\right]$. We identify any two $G$-morphisms that agree $\mu$-almost everywhere and write $\operatorname{Mor}_{G}(X, Y)$ for the set of equivalence classes. We shall distinguish a subset of morphisms

$$
\operatorname{Mor}_{G}^{1}(X, Y)=\left\{p \in \operatorname{Mor}_{G}(X, Y): p \text { relatively p.m.p. }\right\} \text {. }
$$

Recall that given $X \in \mathcal{M} \mathcal{P} \mathcal{P}_{G}$ and $Z \in \mathscr{B}_{G}$ we denote by 


$$
\operatorname{Map}_{G}(X, Z)
$$

the set of equivalence classes of Borel $G$-equivariant maps $\Phi: X \rightarrow Z$.

Let $H$ be a closed subgroup of $G$. Then every Borel $G$-space is a Borel $H$-space, and every Borel $G$-map is a Borel $H$-map. This holds similarly for the measurable category. Hence, we have the restriction functor

$$
\operatorname{Res}_{H}^{G}: \mathscr{B}_{G} \rightarrow \mathcal{B}_{H}, \quad \operatorname{Res}_{H}^{G}: \mathcal{M} \mathcal{M} \mathcal{P}_{G} \rightarrow \mathcal{M} \mathcal{M P} \mathcal{P}_{H}
$$

There is also a natural construction of the induction functor (cf. [33, Section 4.2])

$$
\operatorname{Ind}_{H}^{G}: \mathscr{B}_{H} \rightarrow \mathscr{B}_{G}, \quad \operatorname{Ind}_{H}^{G}: \mathcal{M} \mathcal{M} \mathcal{P}_{H} \rightarrow \mathcal{M} \mathcal{M P} G
$$

defined as follows. Given a Borel $H$-space $X$, consider the factor map $\pi: G \times X \rightarrow$ $(G \times X) / \sim$ where $\sim$ is the equivalence relation $(g, x) \sim\left(g h, h^{-1} x\right)$ for $g \in G$, $h \in H$, and $x \in X$. As a measure space, $(G \times X) / \sim$ is isomorphic to $G / H \times X$ and consequently is a standard Borel space. The $G$-action on $(G \times X) / \sim$ is given by $g\left[g_{1}, x\right]=\left[g g_{1}, x\right]$ for $g, g_{1} \in G$ and $x \in X$. This defines the induced $G$-space $\operatorname{Ind}_{H}^{G}(X)$. Given an $H$-map $p: X \rightarrow Y$ between Borel $H$-spaces, define the induced map

$$
\operatorname{Ind}_{H}^{G}(p): \operatorname{Ind}_{H}^{G}(X) \rightarrow \operatorname{Ind}_{H}^{G}(Y)
$$

by $[g, x] \mapsto[g, p(x)]$ for $g \in G$ and $x \in X$. In the measurable category, one applies the same constructions to the underlying Borel spaces. As for the measures, let $\lambda$ be a probability measure on $G$ equivalent to the Haar measure on $G$. We equip $(G \times X) / \sim$ with the probability measure obtained by pushing forward $\lambda \times \mu$ under the quotient map $G \times X \rightarrow(G \times X) / \sim$. The $G$-action preserves the induced measure class on $\operatorname{Ind}_{H}^{G}(X)$. We leave the proof of the following straightforward statement to the reader.

\section{LEMMA 2.1}

Let $X, Y \in \mathcal{M} \in \mathcal{P}_{H}$.

(a) If $p \in \operatorname{Mor}_{H}(X, Y)$, then $\operatorname{Ind}_{H}^{G}(p) \in \operatorname{Mor}_{G}\left(\operatorname{Ind}_{H}^{G}(X), \operatorname{Ind}_{H}^{G}(Y)\right)$.

(b) If $p \in \operatorname{Mor}_{H}^{1}(X, Y)$, then $\operatorname{Ind}_{H}^{G}(p) \in \operatorname{Mor}_{G}^{1}\left(\operatorname{Ind}_{H}^{G}(X), \operatorname{Ind}_{H}^{G}(Y)\right)$.

Next we show that the functors $\operatorname{Res}_{H}^{G}$ and $\operatorname{Ind}_{H}^{G}$ are formally adjoint.

PROPOSITION 2.2 (Frobenius reciprocity)

Let $X \in \mathcal{M} \mathcal{M} \mathcal{P}_{H}, Y \in \mathcal{M} \mathcal{C P} \mathcal{P}_{G}$, and $Z \in \mathscr{B}_{G}$. Then there are natural bijections

$$
\begin{aligned}
& \operatorname{Mor}_{H}\left(X, \operatorname{Res}_{H}^{G}(Y)\right) \simeq \operatorname{Mor}_{G}\left(\operatorname{Ind}_{H}^{G}(X), Y\right), \\
& \operatorname{Mor}_{H}^{1}\left(X, \operatorname{Res}_{H}^{G}(Y)\right) \simeq \operatorname{Mor}_{G}^{1}\left(\operatorname{Ind}_{H}^{G}(X), Y\right), \\
& \operatorname{Map}_{H}\left(X, \operatorname{Res}_{H}^{G}(Z)\right) \simeq \operatorname{Map}_{G}\left(\operatorname{Ind}_{H}^{G}(X), Z\right),
\end{aligned}
$$


in all three cases given by

$$
\Phi \longmapsto([g, x] \mapsto g \Phi(x))
$$

\section{Proof}

Let $\Phi: X \rightarrow Y$ be an $H$-map, where $Y$ is a $G$-space. It follows from [33, Proposition B.5] that after modifying $\Phi$ on a null set, there exists an $H$-invariant Borel subset $X_{0} \subset X$ of full measure such that $\left.\Phi\right|_{X_{0}}$ is $H$-equivariant. This shows that (2.1) gives a well-defined map from $\operatorname{Mor}_{H}\left(X, \operatorname{Res}_{H}^{G}(Y)\right)$ to $\operatorname{Mor}_{G}\left(\operatorname{Ind}_{H}^{G}(X), Y\right)$.

Now we construct its inverse. Every element in $\operatorname{Mor}_{G}\left(\operatorname{Ind}_{H}^{G}(X), Y\right)$ can be lifted to a map $\Psi: G \times X \rightarrow Y$ such that

$$
\Psi\left(g g^{\prime}, x\right)=g \Psi\left(g^{\prime}, x\right) \quad \text { and } \quad \Psi\left(g^{\prime} h^{-1}, h \cdot x\right)=\Psi\left(g^{\prime}, x\right)
$$

for every $g \in G, h \in H$, and almost every $\left(g^{\prime}, x\right) \in G \times X$. Moreover, this correspondence respects the measure class-preserving property. Applying [33, Proposition B.5] to the action of $G \times H$ on $G \times X$ given by

$$
(g, h) \cdot\left(g^{\prime}, x\right)=\left(g g^{\prime} h^{-1}, h \cdot x\right),
$$

we conclude that, after modifying $\Psi$ on a set of measure zero, we may assume that there exists an $H$-invariant Borel subset $X_{0}$ of $X$ with full measure such that (2.2) holds for all $\left(g^{\prime}, x\right) \in G \times X_{0}$. Then $\Phi(x)=\Psi(e, x), x \in X$, is an $H$-map and it is clear that it defines the inverse to the map (2.1).

The induced space $\operatorname{Ind}_{H}^{G}(X)$ is easy to describe for $G$-spaces.

PROPOSITION 2.3

For every $X \in \mathcal{M} C \mathcal{P}_{G}$,

$$
\operatorname{Ind}_{H}^{G}\left(\operatorname{Res}_{H}^{G}(X)\right) \simeq G / H \times X
$$

where the latter space is equipped with the product measure class and the diagonal action.

\section{Proof}

The map $G \times X \rightarrow G \times X:(g, x) \mapsto(g, g \cdot x)$ induces an isomorphism of the spaces in question.

Now we establish several corollaries that give correspondences among sets of maps in different categories. 
COROLLARY 2.4

Let $X, Y \in \mathcal{M} \mathcal{P} \mathcal{P}_{G}$, let $Z \in \mathscr{B}_{G}$, and let $H<G$ be a closed subgroup. Then, under the bijections given by Propositions 2.2 and 2.3,

$$
\begin{aligned}
\operatorname{Mor}_{H}(X, Y) \simeq \operatorname{Mor}_{G}(G / H \times X, Y), \\
\operatorname{Mor}_{H}^{1}(X, Y) \simeq \operatorname{Mor}_{G}^{1}(G / H \times X, Y), \\
\operatorname{Map}_{H}(X, Z) \simeq \operatorname{Map}_{G}(G / H \times X, Z) .
\end{aligned}
$$

\section{Proof}

The claim follows from Propositions 2.2 and 2.3.

COROLLARY 2.5

Let $H, L<G$ be closed subgroups, let $Y \in \mathcal{M} \mathcal{M} \mathcal{P}_{G}$, and let $Z \in \mathcal{B}_{G}$. Then there are natural bijections

$$
\begin{aligned}
& \operatorname{Mor}_{L}(G / H, Y) \simeq \operatorname{Mor}_{H}(G / L, Y), \\
& \operatorname{Mor}_{L}^{1}(G / H, Y) \simeq \operatorname{Mor}_{H}^{1}(G / L, Y), \\
& \operatorname{Map}_{L}(G / H, Z) \simeq \operatorname{Map}_{H}(G / L, Z)
\end{aligned}
$$

\section{Proof}

Indeed, $\operatorname{Map}_{L}(G / H, Z) \simeq \operatorname{Map}_{G}(G / L \times G / H, Z) \simeq \operatorname{Map}_{H}(G / L, Z)$ using Corollary 2.4. This holds similarly for the other maps.

\section{Remark 2.6}

Let us record the correspondence $\operatorname{Map}_{L}(G / H, Z) \simeq \operatorname{Map}_{H}(G / L, Z)$ explicitly. Denote $Y=G / L$, and denote by $\sigma: Y \rightarrow G$ a Borel cross-section of the projection $g \mapsto g L$. Then the map $\ell: G \times Y \rightarrow G$ defined by

$$
\ell(g, y)=\sigma(g \cdot y)^{-1} g \sigma(y)
$$

takes values in $L$, and forms a Borel cocycle. Given an $L$-map $f: G / H \rightarrow Z$ we define $F: G / L \rightarrow Z$ by

$$
F(y)=\sigma(y) \cdot f\left(\sigma(y)^{-1} H\right)
$$

and observe that for $h \in H$ one has, using the $L$-equivariance of $f$,

$$
\begin{aligned}
F(h \cdot y) & =\sigma(h \cdot y) \cdot f\left(\sigma(h \cdot y)^{-1} H\right) \\
& =\sigma(h \cdot y) \cdot f\left(\ell(h, y) \sigma(y)^{-1} H\right)=\sigma(h \cdot y) \ell(h, y) \cdot f\left(\sigma(y)^{-1} H\right) \\
& =\sigma(h \cdot y) \ell(h, y) \sigma(y)^{-1} \cdot F(y)=h \cdot F(y) .
\end{aligned}
$$


The correspondence $f \mapsto F$ is an explicit identification of $\operatorname{Map}_{L}(G / H, Z)$ with $\operatorname{Map}_{H}(G / L, Z)$, depending on $\sigma: G / L \rightarrow G$.

For our purposes it will suffice to consider maps from measurable spaces to Borel spaces. Hence we state the following corollary for this case only, others being analogous.

COROLLARY 2.7

Let $H, L, M$ be closed subgroups of $G$ with $L<M$, and let $Z \in \mathscr{B}_{G}$. Then the natural inclusion map

$$
\operatorname{Map}_{M}(G / H, Z) \hookrightarrow \operatorname{Map}_{L}(G / H, Z)
$$

is a bijection if and only if the natural map

$$
\operatorname{Map}_{H}(G / M, Z) \rightarrow \operatorname{Map}_{H}(G / L, Z),
$$

induced by $G / L \rightarrow G / M$, is a bijection.

Proof

It is straightforward to check that one has the commutative diagram

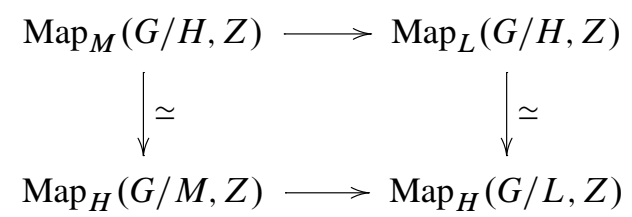

where the vertical arrows are the bijections given by Corollary 2.5. The claim follows.

\section{Borel density theorem}

Fix a finite set $S$ and a collection of nondiscrete local fields $k_{v}, v \in S$, of zero characteristic. Let $V=\prod_{v \in S} \mathbf{V}_{v}\left(k_{v}\right)$ be an $S$-variety. It carries the Zariski topology, by which we mean the product of the Zariski topologies of the factors $\mathbf{V}_{v}, v \in S$, and the analytic topology coming from the analytic structures of the local fields. An $S$-algebraic quotient $H \rightarrow K$ of an $S$-algebraic group $H$ is called compact if $K$ is compact in the analytic topology.

For an $S$-algebraic variety $V$, we set

$$
\operatorname{Prob}^{0}(V)=V, \quad \operatorname{Prob}^{n+1}(V)=\operatorname{Prob}\left(\operatorname{Prob}^{n}(V)\right) \quad(n \geq 0) .
$$

We shall need the following version of the Borel density theorem. 
THEOREM 3.1 (Borel density)

Let $H$ be an $S$-algebraic group with no compact $S$-algebraic factors, and let $V$ be an $S$-algebraic $H$-space. Then, for all $n \geq 0$,

$$
\operatorname{Prob}^{n}(V)^{H}=\operatorname{Prob}^{n}\left(V^{H}\right) \text {. }
$$

Note that the existence of $H$-invariant measures implies the existence of $H$-fixed points. The set of $H$-fixed points $V^{H}$ is the product of $k_{v}$-points of fixed-point varieties

$$
V^{H}=\prod_{v \in S} \mathbf{V}_{v}^{\mathbf{H}_{v}}\left(k_{v}\right)
$$

\section{Proof}

The inclusion $\operatorname{Prob}^{n}(V)^{H} \supset \operatorname{Prob}^{n}\left(V^{H}\right)$ is trivial. We shall prove the other inclusion by induction on $n$, starting with $n=1$. When $V=\mathbf{V}_{v}\left(k_{v}\right)$ is an algebraic variety defined over a single local field $k_{v}$, the claim is well known (see, for instance, [27, Theorem 3.9]). To handle the general $S$-algebraic case, consider the projections $p_{v}$ : $V \rightarrow \mathbf{V}_{v}\left(k_{v}\right), v \in S$. For $\mu \in \operatorname{Prob}(V)^{H}$, the pushforward measures satisfy

$$
p_{v *}(\mu) \in \operatorname{Prob}\left(\mathbf{V}_{v}\left(k_{v}\right)\right)^{\mathbf{H}_{v}\left(k_{v}\right)}=\operatorname{Prob}\left(\mathbf{V}_{v}\left(k_{v}\right)^{\mathbf{H}_{v}\left(k_{v}\right)}\right),
$$

where $H_{v}=\mathbf{H}_{v}\left(k_{v}\right)$. This implies that $\mu$ is supported on $V^{H}=\prod_{v \in S} V_{v}\left(k_{v}\right)^{H_{v}}$, as required.

Assume the validity of the theorem for $n \geq 1$. The barycenter map (see [17]) gives an $H$-equivariant map

$$
\text { bar: } \operatorname{Prob}\left(\operatorname{Prob}^{n}(V)\right) \rightarrow \operatorname{Prob}^{n}(V), \quad v \mapsto \int_{\operatorname{Prob}^{n}(V)} \mu d v(\mu) .
$$

By equivariance, invariant measures are mapped to fixed points. Thus, by induction,

$$
\operatorname{bar}\left(\operatorname{Prob}^{n+1}(V)^{H}\right) \subset \operatorname{Prob}^{n}(V)^{H}=\operatorname{Prob}^{n}\left(V^{H}\right) .
$$

If, for $v \in \operatorname{Prob}^{n+1}(V)^{H}$, we have that

$$
v\left(\left\{\mu: \mu\left(\operatorname{Prob}^{n-1}\left(V^{H}\right)\right)<1\right\}\right)>0,
$$

then

$$
\operatorname{bar}(v)\left(\operatorname{Prob}^{n-1}\left(V^{H}\right)\right)=\int_{\operatorname{Prob}^{n}(V)} \mu\left(\operatorname{Prob}^{n-1}\left(V^{H}\right)\right) d \nu(\mu)<1,
$$

which contradicts (3.1). It follows that $v$-almost every measure in $\operatorname{Prob}^{n}(V)$ is supported on $\operatorname{Prob}^{n-1}\left(V^{H}\right)$. Therefore, $v \in \operatorname{Prob}^{n+1}\left(V^{H}\right)$. This completes the proof. 
We recall the notion of discompact radical introduced by Shalom in [27, Proposition 1.4]. The discompact radical $\mathrm{R}_{\mathrm{dc}}(H)$ of an $S$-algebraic group $H$ is the maximal $S$-algebraic subgroup of $H$ which does not have any nontrivial compact $S$-algebraic quotients. We note that $\mathrm{R}_{\mathrm{dc}}(H)$ is normal in $H$, and $H / \mathrm{R}_{\mathrm{dc}}(H)$ is compact (see [27]).

We give two corollaries of the Borel density theorem.

COROLLARY 3.2

Let $H$ be an $S$-algebraic group, and let $V$ be an $S$-algebraic $H$-space. Let $(X, \xi)$ be an $H$-space with an invariant probability measure. Then, for any $H$-map $\Phi: X \rightarrow$ $\operatorname{Prob}^{n}(V)$,

$$
\Phi(x) \in \operatorname{Prob}^{n}\left(V^{\mathrm{R}_{\mathrm{dc}}(H)}\right) \quad \text { for } \xi \text {-a.e. } x \text {. }
$$

In particular, if the $H$-action on $(X, \xi)$ is ergodic, then for some $\eta_{0} \in \operatorname{Prob}^{n}(V)$

$$
\Phi(x) \in H \cdot \eta_{0}
$$

for $\xi$-almost every $x \in X$.

Proof

The first assertion follows from Theorem 3.1 applied to the measure $\Phi_{*}(\xi)$ and the group $\mathrm{R}_{\mathrm{dc}}(H)$. To prove the second assertion, observe that $\Phi_{*}(\xi)$ is ergodic with respect to the action of the compact group $H / \mathrm{R}_{\mathrm{dc}}(H)$. Hence it has to be supported on a single orbit.

The following corollary explains the terminology "Borel density theorem."

COROLLARY 3.3

Let $H$ be an $S$-algebraic group, and let $M<H$ be a closed subgroup, so that $H / M$ has a finite $H$-invariant measure. Then $\mathrm{R}_{\mathrm{dc}}(H)$ is contained in the Zariski closure of $M$.

Proof

Let $\bar{M}$ be the Zariski closure of $M$, and let $V=H / \bar{M}$. Applying Corollary 3.2 to the $H$-map $H / \bar{M} \rightarrow V$, we deduce that $\mathrm{R}_{\mathrm{dc}}(H)$ acts trivially on $H / \bar{M}$; hence $\mathrm{R}_{\mathrm{dc}}(H)<\bar{M}$.

\section{Proofs of the basic results}

Following these preparations, we are in position to prove Theorems 1.1 and 1.5. They correspond to the special cases $n=0,1$ of the following result. 


\section{THEOREM 4.1}

Let $G$ be an $S$-algebraic group, let $H$ be an $S$-algebraic subgroup with no compact $S$-algebraic factors, let $\Gamma<G$ be a lattice so that $\Gamma \curvearrowright G / H$ is ergodic, and let $V$ be an $S$-algebraic $G$-space. Then, for every $n=0,1, \ldots$, viewing $\operatorname{Prob}^{n}(V)$ as a Borel $\Gamma$-space and $G / H$ as an ergodic $\Gamma$-space gives that there is a natural isomorphism

$$
\operatorname{Map}_{\Gamma}\left(G / H, \operatorname{Prob}^{n}(V)\right)=\operatorname{Map}_{G}\left(G / H, \operatorname{Prob}^{n}(V)\right) \cong \operatorname{Prob}^{n}\left(V^{H}\right),
$$

where $w \in \operatorname{Prob}^{n}\left(V^{H}\right)$ corresponds to the map $\Phi_{w}(g H)=g . w$.

Proof

Denote $Y=\operatorname{Prob}^{n}(V)$. By Corollary 3.2, we have the bijections

$$
\operatorname{Map}_{H}(G / \Gamma, Y) \simeq Y^{H} \simeq \operatorname{Map}_{H}(G / G, Y)
$$

Hence, it follows from Corollary 2.7 that the inclusion

$$
\operatorname{Map}_{G}(G / H, Y) \hookrightarrow \operatorname{Map}_{\Gamma}(G / H, Y)
$$

is a bijection as well. This means that every $\Gamma$-map $G / H \rightarrow Y$ agrees almost everywhere with a $G$-map. Finally, it is easy to see that every $G$-map is of the given form.

\section{Proof of Theorem 1.3}

Let the $\mu_{y}$ 's be the measures on $G / H$ coming from the disintegration of $\mu$. We may assume that $y \mapsto \mu_{y}$ is a $\Gamma$-map. Applying Theorem 1.5 to the $\Gamma$-map $\phi: G / H \rightarrow$ $\operatorname{Prob}(G / H)$ defined by $\phi(x)=\mu_{p(x)}$, we see that there exists $\nu_{0} \in \operatorname{Prob}(G / H)$, supported on $(G / H)^{H} \cong \mathcal{N}_{G}(H) / H$, such that $\phi(g H)=g v_{0}$. In particular, $\phi$ is a $G$-map. Let $L$ denote the stabilizer of $\nu_{0}$. Since $y \mapsto \mu_{y}$ is injective, $Y$ is identified with its image under $y \mapsto \mu_{y}=\mu_{p(g H)}=g \nu_{0}$; that is, $Y$ is isomorphic to $G / L$, and under this identification, $p$ is given by $g H \mapsto g L$. Since $L<\mathcal{N}_{G}(H)$, we have that $H \triangleleft L$, and since the disintegration of $\mu_{G / H}$ has $L$-invariant probability measures as fiber measures, there is a finite invariant measure on $L / H$. This implies that $L / H$ is compact.

\section{Proof of Theorem 1.4}

As explained in Section 1.3, a relatively p.m.p. joining of $G / H_{1}$ and $G / H_{2}$ gives rise to a $\Gamma$-map $G / H_{1} \rightarrow \operatorname{Prob}\left(G / H_{2}\right)$. By Theorem $1.5,\left(G / H_{2}\right)^{H_{1}}$ is nonempty; that is, $H_{1}$ is contained in a conjugate of $H_{2}$. Reversing the roles of $H_{1}$ and $H_{2}$ we see that $H_{1}$ contains a conjugate of $H_{2}$. Since the $H_{i}$ 's are $S$-algebraic groups, this implies that $H_{1}$ and $H_{2}$ are conjugate. 


\section{The Mautner property}

Let $G$ be a locally compact second countable group, and let $H$ and $L$ be closed subgroups. We say that $(H, L, G)$ has the Mautner property if, for every continuous unitary representation $\pi: G \rightarrow \mathcal{U}(\mathscr{H})$, a vector which is fixed by $H$ is already fixed by $L$, that is,

$$
\mathscr{H}^{\pi(H)} \subset \mathscr{H}^{\pi(L)} .
$$

When $G$ is clear, we will say that the pair $(H, L)$ has the Mautner property. In fact (fixing $G$ and $H$ ), there is a maximal $L$ such that $(H, L, G)$ has the Mautner property. Take the intersection of all fixators of all spaces of the form $\mathscr{H}^{\pi(H)}$ over all unitary $G$-representations $\pi$. We call this maximal $L$ the Mautner envelope of $H$ in $G$, and denote it by $\bar{H}^{\mathrm{MT}}$. The alert reader will note that our notation suppresses the dependence on $G$. Thus $\left(H, \bar{H}^{\mathrm{MT}}, G\right)$ has the Mautner property, and $(H, L, G)$ has the Mautner property if and only if $L<\bar{H}^{\mathrm{MT}}$. In this section, we analyze Mautner envelopes in $S$-algebraic groups. A closed subgroup of an $S$-algebraic group is called f.i.-algebraic if it is a finite-index subgroup of an $S$-algebraic subgroup $L \subset G$. While the Mautner envelope does not have to be normal or f.i.-algebraic in general, we shall show (see Theorem 5.6 below) that it always contains a cocompact subgroup satisfying both of these properties. Before doing so, let us collect some trivial observations.

\section{LEMMA 5.1}

Let $H$ be a closed subgroup in a locally compact second countable group $G$.

(a) $\overline{\{e\}}^{\mathrm{MT}}=\{e\}$.

(b) If $H$ is compact, then $H=\bar{H}^{\mathrm{MT}}$.

(c) If $\bar{H}^{\mathrm{MT}}$ contains a closed subgroup $N$ which is normal in $G$, then

$$
\rho\left(\bar{H}^{\mathrm{MT}}\right)=\overline{\rho(H)}^{\mathrm{MT}},
$$

where $\rho: G \rightarrow G / N$ is the factor map.

(d) If $N$ is a closed normal subgroup of $G$ containing $H$, then $\bar{H}^{\mathrm{MT}}<N$.

\section{Proof}

(b) Let $\pi: G \rightarrow H \backslash G$ be the quotient map. Given $g \notin H$, let $f$ be a continuous compactly supported function on $H \backslash G$ which vanishes on $\pi(g)$ but not on $\pi(e)$. Then $f \circ \pi \in L^{2}(G)$ is $H$-invariant but not $g$-invariant. Note that (a) is a special case of (b).

(c) The inclusion $\subset$ follows from the fact that any $G / N$-representation is also a $G$-representation. For the other inclusion, let $M=\rho^{-1}\left(\overline{\rho(H)}{ }^{M T}\right)$. We need to show that $(H, M)$ has the Mautner property. Since $N<\bar{H}^{M T}$, given any unitary 
$G$-representation $\pi: G \rightarrow \mathcal{U}(\mathscr{H})$, one has

$$
\mathscr{H}^{\pi(H)} \subset \mathscr{H}^{\pi\left(\bar{H}^{M T}\right)} \subset \mathscr{H}^{\pi(N)},
$$

and since $N$ is normal in $G, \mathscr{H}^{\pi(N)}$ is $\pi(G)$-invariant and so induces a unitary $G / N$ representation on $\mathscr{H}^{\pi(N)}$. In this representation, $\overline{\rho(H)}^{M T}$ acts trivially, so $M$ is trivial on $\mathscr{H}^{\pi(N)}$. In particular, $M$ fixes $\mathscr{H}^{\pi(H)}$.

(d) This can be seen by considering the representation $L^{2}(G / N)$.

Let us review some basic properties of $S$-algebraic groups that follow from wellknown properties of algebraic groups over local fields of characteristic zero (see, e.g., [18]).

PROPOSITION 5.2 (cf. Proposition 3.3 and Theorem 6.14 in [18])

Let $\mathbf{X}=\prod_{v \in S} \mathbf{X}_{v}$ be a product of algebraic varieties over local fields with a transitive action of $\mathbf{G}=\prod_{v \in S} \mathbf{G}_{v}$. Then the set $X$ of $S$-points of $\mathbf{X}$ is a union of finitely many $G$-orbits. Each of these orbits is open and closed in $X$.

The following is a special case of Proposition 5.2.

PROPOSITION 5.3

Let $\rho: G \rightarrow H$ be an algebraic homomorphism of $S$-algebraic groups. Then the image $\rho(G)$ is f.i.-algebraic in $H$.

LEMMA 5.4

Let $\mathbf{G}$ be an $S$-algebraic group, and let $G$ be the corresponding locally compact group. For any closed subgroup $M<G$, the collection $\mathcal{N}$ of all subgroups $N<M$ which are normal in $G$ and correspond to Zariski-connected, f.i.-algebraic subgroups in $G$ contains a unique maximal element.

\section{Definition 5.5}

The unique maximal subgroup $N$ of $M$ as above is called the fi.-algebraic kernel of $M$ and is denoted by $\mathrm{N}_{\mathrm{a}}(M)$.

To justify the term, we note that the f.i.-algebraic kernel $\mathrm{N}_{\mathrm{a}}(M)$ of $M$ is the maximal f.i.-algebraic, Zariski-connected subgroup of $G$ that acts trivially on $G / M$.

\section{Proof of Lemma 5.4}

First let us show that a maximal element of $\mathcal{N}$ (with respect to inclusion) must be unique. Let $U, V \in \mathcal{N}$, and denote by $\mathbf{U}, \mathbf{V}$ their Zariski closures in $\mathbf{G}$ and by $\bar{U}, \bar{V}$ 
the corresponding locally compact subgroups in $G$. Then

$$
[\bar{U}: U]<\infty, \quad[\bar{V}: V]<\infty .
$$

Applying Proposition 5.2 to the action of $\bar{U} \times \bar{V}$ on $\overline{U \cdot V}$, we see that $\bar{U} \cdot \bar{V}$ is of finite index in $\overline{U \cdot V}$. Similarly $U V$ is of finite index in $\bar{U} \cdot \bar{V}$. Clearly, $U V$ is normal and contained in $M$; therefore $U V \in \mathcal{N}$.

We now show that a maximal element of $\mathcal{N}$ exists. If $U_{1}<U_{2}<\cdots$ is an ascending chain in $\mathcal{N}$, then the corresponding chain of Zariski closures stabilizes after finitely many steps because the $\bar{U}_{i}$ 's are connected. Since $U_{i}$ has finite index in $\bar{U}_{i}$, this implies that the original chain stabilizes as well.

Below we use results from [15] and [30] to prove the following theorem, which is the main result of this section. We remind the reader that in our setting all fields $k_{v}$ are of characteristic zero.

THEOREM 5.6 (Mautner envelope)

Let $G$ be an $S$-algebraic group, and let $H$ be an $S$-algebraic subgroup of $G$.

(a) $\bar{H}^{\mathrm{MT}} / \mathrm{N}_{\mathrm{a}}\left(\bar{H}^{\mathrm{MT}}\right)$ is a compact group.

(b) $\bar{H}^{\mathrm{MT}} / \mathrm{N}_{\mathrm{a}}\left(\bar{H}^{\mathrm{MT}}\right)$ is a finite group, provided $H$ has no nontrivial compact $S$ algebraic quotients.

Let $\mathbf{H}$ be an algebraic group over a local field $k$, and let $\mathbf{A}<\mathbf{H}$ be a onedimensional connected algebraic subgroup, isomorphic over $k$ either to the additive group $\mathbf{G}_{a}$ or to the multiplicative group $\mathbf{G}_{m}$. We shall say that $A<H$ is a onedimensional $k$-split subgroup of $H$. Given an $S$-algebraic group $\mathbf{H}$, let $H^{\vee}$ be the smallest closed subgroup of the locally compact group $H=\prod_{v \in S} \mathbf{H}_{v}\left(k_{v}\right)$ containing all the one-dimensional connected split subgroups over the relevant local fields. Given an $S$-algebraic group $G$ containing $H$, let $H^{\wedge}$ be the closed normal subgroup of $G$ generated by $H^{\vee}$. (The dependence on $G$ is hidden in this notation.)

Theorem 5.6 will be deduced from the following three propositions that we prove below.

PROPOSITION 5.7

Let $G$ be an $S$-algebraic group, and let $H$ be an $S$-algebraic subgroup of $G$. Then $H^{\wedge}$ is the Mautner envelope of $H^{\vee}$ in $G$.

PROPOSITION 5.8

Let $G$ be an $S$-algebraic group. Then $G^{\vee}$ is a Zariski-dense subgroup of finite index in $\mathrm{R}_{\mathrm{dc}}(G)$. 
PROPOSITION 5.9

Let $G$ be an $S$-algebraic group, and let $H$ be an $S$-algebraic subgroup of $G$. Then $H^{\wedge}$ is f.i.-algebraic and Zariski-connected.

Proof of Theorem 5.6 (assuming Propositions 5.7, 5.8, 5.9)

Let $N=H^{\wedge}$. By Proposition $5.7, N$ is contained in $\bar{H}^{\mathrm{MT}}$. It is clearly normal, and also f.i.-algebraic and Zariski-connected by Proposition 5.9. It follows that $N$ is contained in the f.i.-algebraic kernel $\mathrm{N}_{\mathrm{a}}\left(\bar{H}^{\mathrm{MT}}\right)$ of $\bar{H}^{\mathrm{MT}}$. Hence, in order to establish assertion (a) it suffices to show that $\bar{H}^{\mathrm{MT}} / N$ is compact.

Denote by $\rho: G \rightarrow G / N$ the factor map. By Lemma 5.1(c), $\rho\left(\bar{H}^{\mathrm{MT}}\right)=\overline{\rho(H)}^{\mathrm{MT}}$; thus we need to establish the compactness of $\overline{\rho(H)}^{\mathrm{MT}}$. Since $H^{\vee}<\operatorname{ker}(\rho) \cap H$ and $H / H^{\vee}$ is compact by Proposition 5.8, it follows that $\rho(H)$ is compact. Now Lemma 5.1(b) implies that $\overline{\rho(H)}^{\mathrm{MT}}$ is compact, proving (a).

We now assume that $H$ has no compact $S$-algebraic quotients. By Proposition 5.8, $H^{\vee}$ is Zariski-dense in $H$. Then the Zariski closure $\bar{N}$ of $N$ contains $H$. By Proposition 5.9, $N$ is Zariski-connected and of finite index in $\bar{N}$. Clearly $\bar{N}$ is normal in $G$, so by Lemma $5.1(\mathrm{~d}), \bar{H}^{\mathrm{MT}}$ is contained in $\bar{N}$. Hence, we have the inclusions

$$
N<\mathrm{N}_{\mathrm{a}}\left(\bar{H}^{\mathrm{MT}}\right)<\bar{H}^{\mathrm{MT}}<\bar{N}
$$

that imply claim (b).

\section{Proof of Proposition 5.7}

Let $A=\mathbf{A}(k)$ be a one-dimensional $k$-split algebraic subgroup of $G$. We first show that $\left(A, A^{\wedge}\right)$ has the Mautner property. Note that we may assume without loss of generality that $G$ is Zariski-connected. We need to consider the cases when $\mathbf{A}$ is isomorphic over $k$ to the additive group $\mathbf{G}_{a}$ and the multiplicative group $\mathbf{G}_{m}$.

When $\mathbf{A} \simeq \mathbf{G}_{a}$, it was proved in [15, Proposition 2.1] that there exists a closed normal subgroup $N$ containing $A$ such that $(A, N)$ has the Mautner property. This implies the claim in this case.

When $\mathbf{A} \simeq \mathbf{G}_{m}$, we use the results of Wang [29]. Let $g$ be an element of infinite order in $A$. We define the following subgroups of $G$ :

$$
\begin{aligned}
U^{+} & =\left\{x \in G: g^{n} x g^{-n} \rightarrow e \text { as } n \rightarrow \infty\right\}, \\
U^{-} & =\left\{x \in G: g^{-n} x g^{n} \rightarrow e \text { as } n \rightarrow \infty\right\}, \\
M & =\left\{x \in G: g^{-n} x g^{n} \text { is bounded for } n \in \mathbb{Z}\right\} .
\end{aligned}
$$

It was shown in [29, Section 2] that

(1) $\quad U^{+}, U^{-}$and $M$ are the $k$-points of Zariski-connected $k$-algebraic subgroups; 
(2) $U^{+}, U^{-}$and $M$ generate $G$;

(3) the closed subgroup $W=\overline{\left\langle U^{+}, U^{-}\right\rangle}$is normal in $G$; and

(4) the pair $(C, W)$ has the Mautner property, where $C=\overline{\langle g\rangle}$.

We claim, in addition, that

(5) $\quad M$ commutes with $A$.

For this we recall the construction of $M$ from [29]. Let $\mathbf{S}$ be the maximal $k$-split torus in $\mathbf{G}$ containing $\mathbf{A}$, and let $\Delta$ be a set of simple roots on $\mathbf{S}$ such that $|\alpha(g)| \leq 1$ for $\alpha \in \Delta$, where $|\cdot|$ denotes the absolute value of $k$. Let $\Theta=\{\alpha \in \Delta:|\alpha(g)|=1\}$, and let $\mathbf{S}_{\Theta}$ be the connected component of the identity in $\bigcap_{\alpha \in \Theta} \operatorname{ker}(\alpha)$. Then the subgroup $\mathbf{M}$ is precisely the centralizer of $\mathbf{S}_{\Theta}$ in $G$. Every $\alpha \in \Theta$ defines a $k$-character of $\mathbf{A}$ such that $\alpha$ is bounded on $A=\mathbf{A}(k)$. Hence, it follows that $\alpha(A)=1$ for every $\alpha \in \Theta$, and $A \subset \mathbf{S}_{\Theta}(k)$. Therefore $M$ commutes with $A$, as claimed.

Let $N=\overline{A W}$, where $W$ is as in (3). Since $M$ commutes with $A$, it follows from (2) that $N$ is normal in $G$. By (4), $(A, N)$ has the Mautner property. Hence, $\left(A, A^{\wedge}\right)$ has the Mautner property as well. This proves our claim for the case $\mathbf{A} \simeq \mathbf{G}_{m}$.

To finish the proof of the proposition, we recall that $H^{\vee}$ is the closed subgroup generated by the one-dimensional split subgroups $A$ of $H$, and we observe that $H^{\wedge}$ is the closed subgroup generated by the corresponding groups $A^{\wedge}$. Since the pairs $\left(A, A^{\wedge}\right)$ have the Mautner property, it follows that the pair $\left(H^{\vee}, H^{\wedge}\right)$ has the Mautner property as well. As $H^{\wedge}$ is normal in $G$, we conclude that it is the Mautner envelope of $H^{\vee}$ by Lemma 5.1(d).

The proof of Proposition 5.8 and later arguments will require the notion of the Lie algebra and exponential map for $S$-algebraic groups, which we now recall. Given an $S$-algebraic group $G$, we introduce its Lie algebra $\operatorname{Lie}(G)$. It is defined to be $\prod_{v \in S} \operatorname{Lie}\left(\mathbf{G}_{v}\right)$ where $\operatorname{Lie}\left(\mathbf{G}_{v}\right)$ is the Lie algebra of the $v$ th local factor. Since each group $\mathbf{G}_{v}$ is defined over $k_{v}$, its Lie algebra has a $k_{v}$-structure, and we consider $\operatorname{Lie}\left(\mathbf{G}_{v}\right)$ as a Lie algebra over $k_{v}$. The notion of the Lie algebra can be defined for any closed subgroup $H$ of $G=\prod_{v \in S} \mathbf{G}_{v}\left(k_{v}\right)$. For this purpose we may assume without loss of generality that all the local fields in $S$ are incompatible (i.e., have different characteristics of the residue fields). Then there exists an open normal subgroup of $H$ that splits as a product of local factors (see, e.g., [21, Proposition 1.5]). We define the Lie algebra of $H$ as the product of the Lie algebras of local factors. The exponential map exp : $\operatorname{Lie}(H) \rightarrow H$ is the product of exponential maps of the local factors, and it defines a diffeomorphism in a neighborhood of zero.

\section{Proof of Proposition 5.8}

It suffices to prove the claim when $\mathbf{G}$ is a connected algebraic group defined over a local field $k$. Let $R=G^{\vee}$. Consider the factor map $\pi: \mathbf{G} \rightarrow \mathbf{G} / \mathrm{R}_{\mathrm{dc}}(\mathbf{G})$. By Proposi- 
tion 5.3, for a one-dimensional split group $A$, the group $\pi(A)$ is f.i.-algebraic and hence compact. Then $\operatorname{ker}(\pi) \cap A$ is infinite, and since $A$ is one-dimensional and connected, $\operatorname{ker}(\pi) \cap A$ is Zariski-dense in $A$. This implies that $\pi(A)=1$. Hence, $R \subset \mathrm{R}_{\mathrm{dc}}(G)$.

Since a discompact group has no f.i.-algebraic subgroups, it suffices to show that $R$ has finite index in $\mathrm{R}_{\mathrm{dc}}(G)$. We may assume, without loss of generality, that $G=$ $\mathrm{R}_{\mathrm{dc}}(G)$. Since the field $k$ has characteristic zero, the group $\mathbf{G}$ has Levi decomposition

$$
\mathbf{G}=\mathbf{S T U}
$$

where $\mathbf{S}$ is a connected semisimple subgroup defined over $k, \mathbf{T}$ is an algebraic torus defined over $k$ that commutes with $\mathbf{S}$, and $\mathbf{U}$ is a normal unipotent subgroup defined over $k$. Since $G=\mathrm{R}_{\mathrm{dc}}(G)$, it follows from [18, Theorem 3.1] that the reductive group ST has no nontrivial $k$-anisotropic quotients.

In particular, this implies that the torus $\mathbf{T}$ is $k$-split. Then $\mathbf{T}$ is isomorphic over $k$ to $\mathbf{G}_{m}^{d}$ and $T \subset R$. Similarly, $U \subset R$; indeed, since $U$ is unipotent, the exponential map is a polynomial isomorphism $\operatorname{Lie}(U) \rightarrow U$. It follows that every element of $U$ is contained in a one-dimensional split unipotent subgroup of the form $\exp (t x)$, $x \in \operatorname{Lie}(U)$.

Let $S^{+}$denote the closed subgroup of $S$ generated by unipotent split subgroups of $S$. Since $S$ does not have any $k$-anisotropic quotients, the subgroup $S^{+}$has finite index in $S$ by [18, Section 7.2]. It follows that $R \cap S T U$ has finite index in $S T U$. Since $G$ is a homogeneous space with respect to the action of $S \times T \times U$ given by $(s, t, u) \cdot g=s t g u^{-1}$, it follows from Proposition 5.2 that $G$ is a finite union of double cosets of $(S T, U)$. Using that $U$ is normal, we conclude that $S T U$ is a finite-index subgroup of $G$. This implies the claim.

Proposition 5.9 will be deduced from the following more general theorem.

\section{THEOREM 5.10}

Let $\mathbf{G}$ be an algebraic group defined over a local field $k$, and let $\mathbf{A}_{i}, i \in I$, be a family of connected $k$-subgroups closed under conjugation by elements of $G$. Then the closed subgroup $N$ of $G$ generated by $A_{i}, i \in I$, is f.i.-algebraic.

\section{Proof of Proposition 5.9 (assuming Theorem 5.10)}

Note that $H^{\wedge}$ is generated by all the conjugates of the one-dimensional split $S$ algebraic subgroups of $H$. By Theorem 5.10, it is f.i.-algebraic, and it is Zariskiconnected since it is generated by Zariski-connected subgroups.

The rest of this section will be devoted to the proof of Theorem 5.10. Our first step toward the proof of Theorem 5.10 is the case of a solvable group. 
LEMMA 5.11

Let $\mathbf{G}$ be a solvable algebraic group defined over a local field $k$, and let $\mathbf{A}_{i}, i \in I$, be a family of connected $k$-algebraic subgroups. Suppose that there is no proper normal algebraic subgroup of $\mathbf{G}$ containing all of the $\mathbf{A}_{i}$ 's. For each $i$ let $H_{i}$ be a finite-index subgroup of $A_{i}$. Let $N$ be a closed subgroup of $G$ which is normal in a finite-index subgroup of $G$ and which contains all of the $H_{i}$ 's. Then $N$ is of finite index in $G$.

\section{Proof}

The group $\mathbf{G}$ must be connected, as it is generated by the connected groups $\mathbf{A}_{i}$ and their conjugates. The group $N$ is Zariski-dense in $\mathbf{G}$ since it is normalized by a Zariski-dense subgroup and its Zariski closure contains all of the $\mathbf{A}_{i}$ 's. By dimension considerations we may assume that the collection $I$ is finite. When $\mathbf{G}$ is abelian, the lemma follows from Proposition 5.3 by considering the homomorphism $\prod_{I} \mathbf{A}_{i} \rightarrow \mathbf{G}$.

For the general case, we will proceed by induction on $\operatorname{dim} \mathbf{G}$. We will show that G contains a normal algebraic subgroup $\mathbf{M}$ such that $M \cap N$ is of finite index in $M$; from this, the statement will follow by applying the induction hypothesis to $\mathbf{G} / \mathbf{M}$. We will use the notation of [5], and let $\mathscr{D}^{i}(\mathbf{G})$ denote the subgroups in the derived series of $\mathbf{G}$. We will focus on the last nontrivial group $\mathbf{D}$ in this series, and distinguish two cases, according to whether $\mathbf{D}$ is or is not central in $\mathbf{G}$.

If $\mathbf{D}$ is not central in $\mathbf{G}$, then since $N$ is Zariski-dense in $\mathbf{G}$, there exists $n_{0} \in N$ which does not centralize $\mathbf{D}$. Consider the map $\varphi=\varphi_{n_{0}}$, where $\varphi_{n}: \mathbf{D} \rightarrow \mathbf{D}$ is defined by $x \mapsto[n, x]=n x n^{-1} x^{-1}$. Using the fact that $\mathbf{D}$ is abelian we obtain the formula

$$
[n, x y]=[n, x][n, y]^{x}=[n, x][n, y]
$$

that is, $\varphi$ is a $k$-algebraic homomorphism. Denote the Zariski closure of the image by $\mathbf{L}$. Applying Proposition 5.3 we find that $\varphi(D)$ is of finite index in $L$. On the other hand, whenever $x \in N_{G}(N), \varphi(x) \in N$. Since $N_{G}(N)$ is of finite index in $G$, we also have that $N_{D}(N)$ is of finite index in $D$. This implies that $L \cap N$ is of finite index in $L$, and hence for any $n \in N$, the conjugate $L^{n}$ also satisfies that $L^{n} \cap N$ is of finite index in $L^{n}$. Let $\mathbf{M}$ be the smallest $k$-algebraic group containing all the conjugates $\mathbf{L}^{n}$, for $n \in N$. Each $L^{n}$ is contained in $\mathbf{D}$ since $\mathbf{D}$ is normal in $\mathbf{G}$. Thus $\mathbf{M}$ is contained in $\mathbf{D}$ and hence abelian. Since $N$ is Zariski-dense, $\mathbf{M}$ is a normal subgroup of $\mathbf{G}$. Since the theorem is already proved for abelian groups, we now apply it to the groups $\mathbf{M}$ (in place of $\mathbf{G}$ ) and $L^{n}$ (in place of $H_{i}$ ) to obtain that $M \cap N$ is of finite index in $M$, completing the proof in this case.

If $\mathbf{D}$ is central in $\mathbf{G}$, then we let $\mathbf{E}$ be the preceding term in the derived series; that is, $\mathbf{D}=(\mathbf{E}, \mathbf{E})$. For each $n \in N$ we can define $\varphi_{n}$ as in the previous case. Using [5, Proposition 2.2], an induction shows that, for each $i, N \cap D^{i}(\mathbf{G})$ is Zariski-dense in $\mathscr{D}^{i}(\mathbf{G})$. In particular, we can find $n \in N \cap E$ such that $\varphi_{n}(E)$ is nontrivial. We con- 
sider $\varphi=\varphi_{n}$ as a $k$-algebraic map $\mathbf{E} \rightarrow \mathbf{D}$. Since $\mathbf{D}$ is central in $\mathbf{G},(5.1)$ shows that $\varphi$ is a homomorphism in this case as well. Now the same argument can be repeated.

\section{LEMMA 5.12}

Let $\mathbf{G}$ be a connected $k$-algebraic group with no solvable nontrivial quotients. Let $N$ be an open normal subgroup of $G$. Then $N$ is of finite index in $G$.

\section{Proof}

We denote the unipotent radical of $\mathbf{G}$ by $\mathbf{U}$, and let $\mathbf{G}=\mathbf{S} \ltimes \mathbf{U}$ be a Levi decomposition. The subgroup $\mathbf{S}$ is semisimple. (Otherwise there is an abelian quotient.) By passing to a covering group, we may and will assume that $\mathbf{S}$ is simply connected. Since $N$ is open in $G$, it is enough to prove that $N$ is cocompact in $G$. By [14, Corollary 2.3.2(a)], $N$ contains the group $S^{\prime}$ where $\mathbf{S}^{\prime}<\mathbf{S}$ is the subgroup consisting of the product of all $k$-isotropic factors. Since $S^{\prime} U$ is cocompact in $G$, there is no loss of generality in assuming that $\mathbf{S}^{\prime}=\mathbf{S}$ and hence $S<N$. For $\mathbf{U}$ trivial, the proof is now complete. For the general case, we proceed by induction on the dimension of $\mathbf{U}$.

Since $\mathbf{S}^{\prime}$ is generated by unipotent subgroups and is not normal in $\mathbf{G}$ (otherwise there will be a solvable quotient), there exists a connected unipotent subgroup $\mathbf{V}<\mathbf{S}$ which is not normalized by $\mathbf{U}$. Since $U$ is Zariski-dense in $\mathbf{U}$, we can find $u \in U$ which does not normalize $\mathbf{V}$. Consider the group $\mathbf{W}$ generated by $\mathbf{V}$ and $\mathbf{V}^{u}$. It is a subgroup of $\mathbf{V U}$ and hence unipotent. Then $V$ and $V^{u}$ are contained in $N$, and hence by Lemma 5.11, $N$ contains a finite-index subgroup of $W$. Let $\mathbf{H}_{1}$ denote $\mathbf{W} \cap \mathbf{U}$. This is a connected group (since $\mathbf{U}$ is unipotent) which has positive dimension in $\mathbf{U}$. Then $N$ contains a finite-index subgroup of $H_{1}$. Consider all the conjugations of $H_{1}$ by elements of $N$. These conjugates generate a subgroup $\mathbf{H}$ which is normal in $\mathbf{G}$. By another application of Lemma 5.11, $N$ contains a finite-index subgroup of $\mathbf{H}$. The proof now follows by applying the induction hypothesis to the group $\mathbf{G} / \mathbf{H}$.

\section{Proof of Theorem 5.10}

Replacing $\mathbf{G}$ by the algebraic subgroups generated by the groups $\mathbf{A}_{i}$, we need to prove that $N$ is of finite index in $G$. The group $N$ is clearly normal in $G$ and Zariskidense. The group $\mathbf{G}$ must be Zariski-connected, as it is generated by the connected groups $\mathbf{A}_{i}$. By dimension considerations, we can replace $I$ by a finite subset, so that $\mathbf{G}$ is still generated by the $\mathbf{A}_{i}$ 's. By [5, Proposition 2.2] and [14, Theorem 2.5.3(2)], $N<G$ is open. Let $\mathbf{G}_{0}$ be the smallest normal subgroup of $\mathbf{G}$ containing all the semisimple subgroups of $\mathbf{G}$, and let $\mathbf{G}_{1}=\mathbf{G} / \mathbf{G}_{0}$. Then $N_{0}=N \cap G_{0}$ is open in $G_{0}$ and consequently of finite index by Lemma 5.12. By Lemma 5.11, the image of $N$ in $G_{1}$ is of finite index. This implies that $N$ is of finite index in $G$. 


\section{The relative Borel density theorem}

\subsection{Relative Borel density}

In this section we will state and prove the relative Borel density theorem (Theorem 6.2).

\section{Definition 6.1}

Let $G$ be an $S$-algebraic group, and let $L$ be a closed subgroup. A fat complement for $L$ in $G$ is a subgroup $M<G$ satisfying the following three conditions:

(F1) $L \cdot M=G$,

(F2) the Zariski closure of $M$ contains the discompact radical $\mathrm{R}_{\mathrm{dc}}(G)$, and

(F3) $\quad \mathrm{N}_{\mathrm{a}}(M) \cap L$ is cocompact in $L$.

THEOREM 6.2 (Relative Borel density)

Let $G$ be an $S$-algebraic group, and let $H$ be an $S$-algebraic subgroup of $G$. Let $(X, \xi)$ be a $G$-space with an invariant probability measure which is ergodic for the action of $H$. Then there exist a closed subgroup $M$ of $G$ that is cocompact, has finite covolume, and is a fat complement for $H$ in $G$, and a measure-preserving $G$-map $\pi: X \rightarrow G / M$ such that the following holds.

For every $S$-algebraic $G$-space $V$, for every $n \geq 0$, and for every $H$-map $i$ : $X \rightarrow \operatorname{Prob}^{n}(V)$, there exists an $H$-map $j: G / M \rightarrow V$ such that $i=j \circ \pi$ almost everywhere. That is, we have the following diagram:

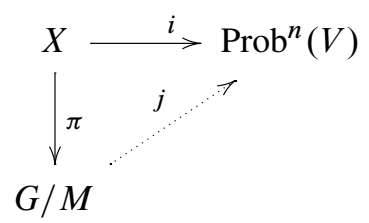

Note that, by property (F3), if $H$ has no compact algebraic factors, then $i$ is constant almost everywhere. We will see below (Corollary 6.3) that if $G$ has no compact $S$-algebraic factors, then $i$ has an essentially finite image.

\section{Proof}

Let $R=\mathrm{R}_{\mathrm{dc}}(H)$. By Corollary 3.2, the image of $i$ is contained in $\operatorname{Prob}^{n}\left(V^{R}\right)$. Let $X / / R$ denote the space of ergodic components for the action of $R$ on $(X, \xi)$, as described in the Appendix. Applying Proposition A.6(a), we find that $i$ factors through $X / / R$. Since $\xi$ is $G$-invariant and finite, Corollary A.7 implies that $X / / R$ is canonically identified with $X / / \bar{R}^{\mathrm{MT}}$. Let $N=\mathrm{N}_{\mathrm{a}}\left(\bar{R}^{\mathrm{MT}}\right)$ be the f.i.-algebraic kernel of the Mautner envelope of $R$ (see Definition 5.5). It follows that $i$ factors through 
$X / / N$, which, by Proposition A.6(b), is a $G$-space on which $N$ acts trivially. Moreover, the factor map $P_{N}: X \rightarrow X / / N$ is a $G$-map.

Since $H$ acts ergodically on $X, H / H \cap N$ acts ergodically on $X / / N$. By Theorem 5.6(b), $N$ is of finite index in the Mautner envelope of $R$. It follows that $N \cap R$ is of finite index in $R$. Since $H / R$ is compact, $H /(H \cap N)$ is compact as well. Because of ergodicity, the measure $\left(P_{N}\right)_{*} \xi$ is supported on a single orbit of $H$. In particular, it follows that $X / / N$ is isomorphic as a $G$-space to $G / M$, where $M$ is a closed cocompact subgroup of $G$ of finite covolume, with $N<M$.

We denote by $\pi: X \rightarrow G / M$ the map corresponding to $P_{N}: X \rightarrow X / / N$. Then the existence of a Borel map $j: G / M \rightarrow Y$ such that $i=j \circ \pi$ follows from Proposition A.6(a). Since $i$ and $\pi$ are $H$-maps, $j$ is an $H$-map as well.

It remains to show that $M$ is a fat complement for $H$ in $G$. Since $H /(H \cap N)$ is compact and acts ergodically on $G / M$, we conclude that $H$ acts transitively on $G / M$ and (F1) follows. To prove (F2), we observe that $G / M$ supports a $G$-invariant measure $\pi(\xi)$. Hence, it follows from Corollary 3.3 that $\mathrm{R}_{\mathrm{dc}}(G)$ is contained in the Zariski closure of $M$. Since $N$ acts trivially on $G / M$, we have that $N<\mathrm{N}_{\mathrm{a}}(M)$. This implies (F3), because $H \cap N$ is cocompact in $H$.

\subsection{Fat complements}

In this section we discuss fat complements. Our goal is the following proposition, which will provide more information on the conclusion of Theorem 6.2.

\section{PROPOSITION 6.3}

Let $G, H$, and $i$ be as in Theorem 6.2. If $G$ has no compact $S$-algebraic factors, then $i$ has finite image.

Proposition 6.3 is an immediate consequence of the following.

\section{THEOREM 6.4}

Let $G$ be an $S$-algebraic group with no compact algebraic quotients. Let $H$ be an $S$-algebraic subgroup, and let $M$ be a fat complement of $H$ in $G$. Then $M$ is a finiteindex subgroup of $G$.

For a Lie algebra $\mathfrak{g}$, we denote by $\mathfrak{g}^{\prime}$ its commutator subalgebra. Let Ad : $G \rightarrow$ $\mathrm{GL}(\operatorname{Lie}(G))$ denote the adjoint representation. We recall that the differential of Ad is given by $\operatorname{ad}(x)=[x, \cdot], x \in \operatorname{Lie}(G)$, and there exists a neighborhood $\mathcal{O}$ of the origin in $\operatorname{Lie}(G)$ such that $\exp : \mathcal{O} \rightarrow G$ is well defined and

$$
\operatorname{Ad}(\exp (x))=\exp (\operatorname{ad}(x)) \quad \text { for all } x \in \mathcal{O} \text {. }
$$


For the proof of Theorem 6.4 we will need the following version of the Malcev lemma, which follows, for example, from [5, Corollary 7.9].

\section{LEMMA 6.5}

Let $G$ be an $S$-algebraic group, let $\mathfrak{g}$ be its Lie algebra, and let $\mathfrak{m}$ be a Lie subalgebra of $\mathfrak{g}$. Suppose that the subgroup $M$ of $G$ generated by $\exp (\mathfrak{m})$ is Zariski-dense in $G$. Then $\mathfrak{g}^{\prime}=\mathfrak{m}^{\prime}$.

\section{Proof of Theorem 6.4}

Let $N=\mathrm{N}_{\mathrm{a}}(M)$. Let $\mathfrak{g}, \mathfrak{h}, \mathfrak{n}$, and $\mathfrak{m}$ denote the Lie algebras corresponding to $G, H$, $N$, and $M$, respectively. The hypothesis that $G$ has no compact factors and (F2) imply that $M$ is Zariski-dense; hence

$$
\operatorname{Ad}(G) \mathfrak{m} \subset \mathfrak{m}
$$

We choose open subgroups $M^{\circ}, N^{\circ}$, and $G^{\circ}$ of $M, N$, and $G$, respectively, such that (1) $\quad G^{\circ}$ normalizes $M^{\circ}$ and $N^{\circ}$, and $N^{\circ}$ normalizes $M^{\circ}$; and

(2) the group $M^{\circ} N^{\circ}$ is generated by open neighborhoods which are in the image of a neighborhood satisfying (6.1).

We observe that one can choose these subgroups as products of local factors (see [24, Proposition 1.5]):

$$
M^{\circ}=\prod_{v} M_{v}^{\circ}, \quad N^{\circ}=\prod_{v} N_{v}^{\circ}, \quad G^{\circ}=\prod_{v} G_{v}^{\circ} .
$$

For archimedean $v$, (2) holds provided that $M_{v}^{\circ}$ and $N_{v}^{\circ}$ are connected. For nonarchimedean $v$, the groups have bases of neighborhoods of identity consisting of Lie subgroups, so that $M_{v}^{\circ}$ and $N_{v}^{\circ}$ can be taken to be sufficiently small to satisfy (2). Property (1) can be satisfied because of (6.2).

Since $G$ is Zariski-connected, the subgroup $G^{\circ}$ is Zariski-dense in $G$. Let $\bar{M}$ be the group of $S$-points of the Zariski closure of $M^{\circ}$. Then $\bar{M}$ is normal in $G$. It follows from (F1) and the Baire category theorem that the set $H M^{\circ}$ is open in $G$. In particular, it is Zariski-dense in $G$. Since the subgroup $H \bar{M}$ is both f.i.-algebraic (by Proposition 5.3) and open in $G$, we conclude that $H \bar{M}$ has finite index in $G$.

The group $N=\mathrm{N}_{\mathrm{a}}(M)$ is f.i.-algebraic and Zariski-connected. Since $N^{\circ}$ is open in $N$, the subgroup $N^{\circ}$ is Zariski-dense in $\bar{N}$. Let $L$ be the Zariski closure of the subgroup $N^{\circ} M^{\circ}$. Clearly, $L$ is normal and $L \supset \bar{N} \bar{M}$. Hence, $H L$ is of finite index in $G$. Since $M$ is a fat complement of $H, H /(H \cap N)$ is compact. Hence, $G / L$ is compact as well. By our assumption on $G$, the group $N^{\circ} M^{\circ}$ is Zariski-dense in $G$. Hence, we may apply Lemma 6.5 with the subgroup $N^{\circ} M^{\circ}$ to conclude that

$$
\mathfrak{g}^{\prime} \subset \mathfrak{n}+\mathfrak{m} \subset \mathfrak{m} .
$$


Let $\mathbf{G}^{\prime}$ denote the (algebraic) commutator subgroup of $\mathbf{G}$, and let $\pi: \mathbf{G} \rightarrow \mathbf{G} /\left(\mathbf{G}^{\prime} \overline{\mathbf{N}}\right)$ denote the corresponding factor map. Since the algebraic group $\mathbf{G} /\left(\mathbf{G}^{\prime} \overline{\mathbf{N}}\right)$ is abelian, it splits as an almost direct product of anisotropic and split subgroups. Moreover, by our assumption on $G$, the anisotropic component is trivial. On the other hand, since $H /(H \cap N)$ is compact, $\pi(H)$ is a compact f.i.-algebraic subgroup. Hence, $H \subset G^{\prime} \bar{N}$. Now using (F1) and (6.3), we obtain that

$$
\mathfrak{g}=\mathfrak{h}+\mathfrak{m} \subset \mathfrak{g}^{\prime}+\mathfrak{n}+\mathfrak{m}=\mathfrak{m} .
$$

This shows that the group $M$ is open in $G$. On the other hand, the homogeneous space $G / M$ is compact. Hence, $G / M$ has to be finite. This completes the proof.

\section{Completion of the proofs}

We will first state and prove a useful corollary of the results of the previous section.

\section{COROLLARY 7.1}

Let $G$ be an $S$-algebraic group, let $H$ be an $S$-algebraic subgroup of $G$, and let $\Gamma$ be a lattice such that $H \curvearrowright G / \Gamma$ is ergodic.

Then there exists a closed subgroup $\Gamma<M<G$, where $M$ is cocompact, of finite covolume in $G$, and a fat complement of $H$ in $G$ such that, for every $S$-algebraic $G$ space $V$, the inclusion map

$$
\operatorname{Map}_{M}\left(G / H, \operatorname{Prob}^{n}(V)\right) \hookrightarrow \operatorname{Map}_{\Gamma}\left(G / H, \operatorname{Prob}^{n}(V)\right)
$$

and the map

$$
\operatorname{Map}_{H}\left(G / M, \operatorname{Prob}^{n}(V)\right) \rightarrow \operatorname{Map}_{H}\left(G / \Gamma, \operatorname{Prob}^{n}(V)\right),
$$

obtained by precomposing maps from $\operatorname{Map}_{H}\left(G / M, \operatorname{Prob}^{n}(V)\right)$ with the projection $G / \Gamma \rightarrow G / M$, are bijections. When $G$ has no nontrivial compact $S$-algebraic factors, $M$ is of finite index in $G$.

Proof

Applying Theorem 6.2 to $X=G / \Gamma$, we obtain a group $M$ and a $G$-map $G / \Gamma \rightarrow$ $G / M$. Replacing $M$ with a conjugate we may assume that $\Gamma<M$. The second bijection is a direct corollary of Theorem 6.2 and the first follows formally from the second using Corollary 2.7 with $L=\Gamma$. The last assertion follows from Proposition 6.3.

\subsection{Proofs of Theorem 1.6 and Corollaries 1.7 and 1.8}

\section{Proof}

Theorem 1.6 is just the first assertion of Corollary 7.1 in the case $n=1$. (Recall the 
bijection $X^{M \cap H} \cong \operatorname{Map}_{H}(G / M, X)$ described in the introduction.) Corollary 1.7 is the case $n=0$, and the deduction of Corollary 1.8 from Theorem 1.6 follows the same steps as the deduction of Theorem 1.4 from Theorem 1.5.

\subsection{Proof of Theorem 1.11}

\section{Proof}

Fix a map $f \in \operatorname{Map}_{\Gamma}(G / \Lambda, G / \Delta)$, where the target $G / \Delta$ is viewed as a Borel $\Gamma$-space. By Corollary 2.5 we have the duality

$$
\operatorname{Map}_{\Gamma}(G / \Lambda, G / \Delta) \cong \operatorname{Map}_{\Lambda}(G / \Gamma, G / \Delta) .
$$

Given $f \in \operatorname{Map}_{\Gamma}(G / \Lambda, G / \Delta)$ let $F: G / \Gamma \rightarrow G / \Delta$ be the corresponding measurable $\Lambda$-equivariant map. As in Remark 2.6 we fix a Borel cross-section $\sigma: Y=G / \Gamma \rightarrow G$ of the projection $G \rightarrow G / \Gamma$, and take

$$
F(y)=\sigma(y) \cdot f\left(\sigma(y)^{-1} \Lambda\right) .
$$

Let $\mu$ denote the probability measure on the space

$$
X=Y \times Z, \quad \text { where } Y=G / \Gamma, Z=G / \Delta,
$$

obtained by pushing the Haar measure $m_{G / \Gamma}$ to the graph of $F$. Since $F$ is a $\Lambda$-map, $\mu$ is invariant under the action of $\operatorname{Diag}(\Lambda)=\{(\lambda, \lambda) \in G \times G: \lambda \in \Lambda\}$ on $X$. Since $\Lambda$ is a Zariski-dense subgroup of $G$, it acts ergodically on $G / \Gamma$, and hence so does the action $\operatorname{Diag}(\Lambda) \curvearrowright(X, \mu)$. Next we want to use one of the assumptions (RS) or (BQ).

Assuming $(R S)$. To start, $\Lambda$ is a lattice in a subgroup of $G$ which is generated by unipotent elements. Note that we do not assume that this subgroup is connected, that is, $\Lambda$ itself may be generated by unipotent elements. In this situation one can apply the results of Ratner [23] and their extension by Shah [26] and Witte [31] to deduce that $\mu$ is $L$-homogeneous, where $L$ is a closed subgroup of $G \times G$ containing $\operatorname{Diag}(\Lambda)$. This means that there exists $x_{0}=\left(g_{1} \Gamma, g_{2} \Delta\right) \in X$ so that

$$
\Sigma=L \cap\left(g_{1} \Gamma g_{1}^{-1} \times g_{2} \Delta g_{2}^{-1}\right)
$$

is a lattice in $L$ and $\mu$ is the pushforward of the Haar measure $m_{L / \Sigma}$ to $L . x_{0} \subset X$. Since $\mu$ projects onto $m_{G / \Gamma}$, it follows that $L$ projects onto $G$. Consider

$$
H=\{g \in G:(e, g) \in L\} .
$$

This is a closed subgroup in $G$. Recall that $\mu$ is supported on the graph of $F$ :

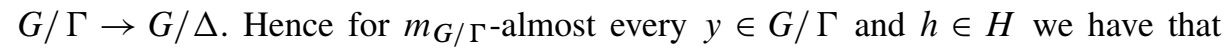
$h . F(y)=F(y)$. Note that given $z=g \Delta \in G / \Delta$ the group $\Delta_{z}=g \Delta g^{-1}$ is defined unambiguously. We have 


$$
H \subset \Delta_{F(y)}
$$

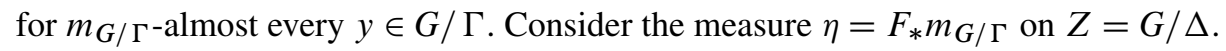
Since $H$ and $\Delta$ are closed sets, there is a conull (with respect to $\eta$ ) $Z_{0} \subset Z$ such that

$$
H<\bigcap_{z \in Z_{0}} \Delta_{z}
$$

Let $M$ denote the projection of $L<G \times G$ to the second factor. Then $\Lambda<M$ and $\eta \in \operatorname{Prob}(Z)$ is $M$-homogeneous. The connected component $M^{0}$ of the identity in $M$ is normal in $M$ and therefore normalized by $\Lambda$, which is Zariski-dense in $G$. Hence $M^{0}$ is normal in $G$. Since $G$ is simple and connected, we have

(1) either $M^{0}=\{e\}$ and $M$ is discrete, or

(2) $M^{0}=M=G$.

Case (1) As $M$ is discrete, $\eta$ is atomic, and since it is a $\Lambda$-invariant and ergodic probability measure, it is supported on a finite $\Lambda$-orbit. That is, $F$ has a finite image. Let $\Lambda_{0}$ be a finite-index normal subgroup of $\Lambda$ acting trivially on the image of $F$. By the Howe-Moore theorem, $\Lambda_{0}$ also acts ergodically on $G / \Gamma$, which implies that $F$ is essentially constant. That is, $\eta=\delta_{z_{0}}$ for some $\Lambda$-fixed point $z_{0}=a \Delta \in G / \Delta$. We deduce $\Lambda \subset \Delta_{z_{0}}=a \Delta a^{-1}$ from (7.2), and have $F(g \Gamma)=y_{0}$ almost everywhere. It follows from (7.1) that

$$
f(g \Lambda)=g a \Delta
$$

Case (2) We have that $M=G$ and $\eta=m_{G / \Delta}$. Since $Z_{0}$ in (7.2) is conull, it follows that $H=\{e\}$. Recalling the definition of $H$, we deduce that

$$
L=\{(g, \rho(g)): g \in G\}
$$

for some continuous homomorphism $\rho: G \rightarrow G$, which is therefore algebraic. As $\operatorname{Diag}(\Lambda)<L$ we get that $\rho(\lambda)=\lambda$ for $\lambda \in \Lambda$, and the Zariski density of $\Lambda$ in $G$ implies that $\rho(g)=g$ for all $g \in G$. Therefore, $F\left(g g_{1} \Gamma\right)=g g_{2} \Delta$ or

$$
F(g \Gamma)=g g_{0} \Delta
$$

with $a=g_{1}^{-1} g_{2}$; in particular, $\Gamma<\Delta_{a}$. From (7.1) we deduce, using a Borel crosssection $s: G / \Lambda \rightarrow G$, that

$$
f(g \Lambda)=s(g \Lambda) F\left(s(g \Lambda)^{-1} \Gamma\right)=s(g \Lambda) s(g \Lambda)^{-1} a \Delta=a \Delta,
$$

which is an almost everywhere constant function. This completes the proof of Theorem 1.11 under assumption (RS). Let us proceed to the proof under assumption (BQ). 
Assuming $(B Q)$. We have that $\Delta<\Delta_{0}$ where $\Delta_{0}$ is a lattice in $G$. Denote by $\pi: G / \Delta \rightarrow G / \Delta_{0}$ the natural projection, and let

$$
F_{0}: G / \Gamma \stackrel{F}{\longrightarrow} G / \Delta \stackrel{\pi}{\longrightarrow} G / \Delta_{0}, \quad \eta_{0}=\pi_{*} \eta \in \operatorname{Prob}\left(G / \Delta_{0}\right) .
$$

Then $\eta_{0}$ is a $\Lambda$-invariant and ergodic probability measure on $G / \Delta_{0}$. Since $\Lambda$ is assumed to be Zariski-dense in $G$, we can apply the recent result of Benoist-Quint [3] to the action $\Lambda \curvearrowright G / \Delta_{0}$ to deduce the dichotomy:

(1) either $\eta_{0}$ is atomic, equidistributed on a finite $\Lambda$-orbit $\Lambda g_{0} \Delta_{0} \subset G / \Delta_{0}$, or

(2) $\eta_{0}=m_{G / \Delta_{0}}$ is the Haar measure on $G / \Delta_{0}$.

In Case (1), $\eta$ is also atomic, and we conclude the proof as in the previous case. We are left with Case (2), where the probability measure $\eta$ on $G / \Delta$ projects onto the normalized Haar measure $m_{G / \Delta_{0}}$ on $G / \Delta_{0}$. We claim that this is possible only if $\Delta$ has finite index in $\Delta_{0}$ and $\eta$ is the normalized Haar measure $m_{G / \Delta}$. (We would like to thank the referee for suggesting the following argument, which is much shorter than our original.)

First observe that since the fibers of $G / \Delta \rightarrow G / \Delta_{0}$ are countable, the probability measure $\eta$ is absolutely continuous with respect to a Haar measure $m_{G / \Delta}$. Indeed, identifying $G / \Delta \cong G / \Delta_{0} \times \Delta_{0} / \Delta$ one can view $\eta$ as

$$
\int_{G / \Delta_{0}} \delta_{x} \times \eta_{x} d m_{G / \Delta_{0}}(x)
$$

for some measurable family $\left\{\eta_{x} \mid x \in G / \Delta_{0}\right\}$ of probability distributions on the countable set $\Delta_{0} / \Delta$, while $m_{G / \Delta}$ corresponds to $m_{G / \Delta_{0}} \times c_{\Delta_{0} / \Delta}$-a product with a multiple of a counting measure on $\Delta_{0} / \Delta$. As $\eta_{x} \ll c_{\Delta_{0} / \Delta}$ it follows that $\eta \ll m_{G / \Delta}$. Since $\eta$ is a probability measure, the Radon-Nikodym derivative $\rho(x)=d \eta / d m_{G / \Delta}(x)$ is a unit vector in $L^{1}(G / \Delta)$, while $f=\sqrt{\rho}$ is a unit vector in $L^{2}(G / \Delta)$. Both $\eta$ and $m_{G / \Delta}$ are $\Lambda$-invariant; hence $f$ is an invariant vector for the unitary $\Lambda$-representation on $L^{2}(G / \Delta)$. This is a restriction of the unitary $G$-representation on $L^{2}(G / \Delta)$. Since $\Lambda$ is not precompact, it follows from the Howe-Moore theorem that $f$ is invariant under $G$. Hence $f$ is constant and $m(G / \Delta)<+\infty$; in other words, $\Delta$ is a lattice in $G$ and $\eta=m_{G / \Delta}$ is the normalized Haar measure on $G / \Delta$.

Now consider the pushforward measure $m$ of $m_{G / \Gamma}$ to the graph of $F: G / \Gamma \rightarrow$ $G / \Delta$. This is a probability measure on

$$
G \times G / \Gamma \times \Delta
$$

invariant under the diagonal action of $\Lambda$. By [3] such a measure should be homogeneous for a subgroup $L<G \times G$ containing $\Lambda$. The argument can now be completed as in the (RS) case by ruling out the possibility that $L=G \times G$ (because $m$ cannot be a product measure $m_{G / \Gamma} \times m_{G / \Delta}$ ) and deducing that the $\Lambda$-equivariant map 
$F: G / \Gamma \rightarrow G / \Delta$ is actually $G$-equivariant. This, in turn, implies that the original $\Gamma$-equivariant map $f: G / \Lambda \rightarrow G / \Delta$ is a constant map.

To prove Theorem 1.9 we use recurrence instead of the existence of a finite invariant measure. An action of a group $A$ on an $A$-space $Z$ is called strictly conservative if, for any nonnull Borel $B \subset Z$ and any compact $C \subset A$, there is $a \in A \backslash C$ such that $a B \cap B$ is nonnull.

\section{LEMMA 7.2}

Let $A$ be a locally compact second countable (lcsc) group, and let $Y$ be a second countable topological space equipped with a Borel probability measure $v$ on which $A$ acts strictly conservatively. Then, for almost every $y \in Y$, there is a sequence $a_{n} \in A$ such that $a_{n} \rightarrow \infty$ and $a_{n} y \rightarrow y$.

\section{Proof}

For an open $U \subset Y$ of positive measure and compact $C \subset A$, let

$$
Y_{C, U}=\{z \in U: \forall a \in A \backslash C, a z \notin U\} .
$$

This is a null set by strict conservativity. Taking an exhaustion of $A$ by countably many compacts $\left\{C_{i}\right\}$ and a countable basis $U_{j}$ of open sets of $Z$, we have that $Y_{\infty}=$ $\bigcup_{i, j: v\left(U_{j}\right)>0} Y_{C_{i}, U_{j}}$ is also a null set, and any point in supp $\backslash Y_{\infty}$ has the required properties.

\section{Proof of Theorem 1.9}

First consider the geodesic flow case, which amounts to the study of $\Gamma$-maps $f$ : $G / C A \rightarrow G / C A$, where the source $G / C A$ is equipped with the Haar measure class and the target $G / C A$ is viewed either as a measured space or as a Borel space. By Corollary 2.5,

$$
\operatorname{Map}_{\Gamma}(G / C A, G / C A) \simeq \operatorname{Map}_{C A}(G / \Gamma, G / C A) \subset \operatorname{Map}_{A}(G / \Gamma, G / C A) .
$$

Let $\phi: G / \Gamma \rightarrow G / C A$ be an $A$-map (actually a $C A$-map) corresponding to the initial $\Gamma$-map $f: G / C A \rightarrow G / C A$. Let $\mu$ be a probability measure in the Haar measure class on $G / \Gamma$, and let $v=\phi_{*} \mu$. Then $v$ is ergodic with respect to the action of $C A$ and hence is $A$-conservative. It follows that for $v$-almost every $x \in G / C A$ there exists a sequence $a_{i} \rightarrow \infty$ in $A$ such that $a_{i} . x \rightarrow x$ (see Lemma 7.2). Identifying $G / C A$ with the space $\partial^{(2)} \mathbf{H}_{k}^{n}$ of pairs of distinct points on the boundary of the symmetric space $\mathbf{H}_{k}^{n}$ of $G$, denote by $\left(\alpha_{-}, \alpha_{+}\right)$the pair of $A$-fixed points on the boundary. It is easy to check that the sequence $a_{i} . x$ may only accumulate to $\left(\alpha_{-}, \alpha_{+}\right)$or to $\left(\alpha_{+}, \alpha_{-}\right)$. Thus for $\mu$-almost every $x \in G / \Gamma$, one has $\phi(x)=\left(\alpha_{-}, \alpha_{+}\right)$or $\left(\alpha_{+}, \alpha_{-}\right)$, and by ergodicity one of these two possibilities occurs almost surely. 
These two cases for $\phi$ correspond to $f$ coinciding almost everywhere with either the identity map or with the flip $F:(\xi, \eta) \mapsto(\eta, \xi)$ of $\partial^{(2)} \mathbf{H}_{k}^{n} \cong G / C A$. Note that $\{\mathrm{Id}, F\}$ is precisely the Weyl group $W_{G}=\mathcal{N}_{G}(A) / \mathcal{Z}_{G}(A)$ of $G$ acting on $G / \mathcal{Z}_{G}=$ $G / C A$ from the right, commuting with the transitive $G$-action (which includes the $\Gamma$-action).

The case of the frame flow corresponds to the study of $\Gamma$-maps $f: G / A \rightarrow G / A$. The reciprocity principle (Corollary 2.5) translates this into the study of an $A$-map $\phi$ : $G / \Gamma \rightarrow G / A$, which by previous discussion is a lift of a constant map $\phi_{0}: G / \Gamma \rightarrow$ $G / C A$ that is either $\phi_{0}(-)=\left(\alpha_{-}, \alpha_{+}\right)$or $\left(\alpha_{+}, \alpha_{-}\right)$. It follows that $\phi(x)=\phi_{0} c_{x}$ for some measurable map $c: G / \Gamma \rightarrow C=\mathcal{Z}_{K}(A)$. From $A$-equivariance we deduce that

$$
\phi_{0} c_{a . x}=\phi(a . x)=a . \phi(x)=a . \phi_{0} c_{x}=\phi_{0} c_{x} \quad(a \in A)
$$

and conclude that $c_{a . x}=c_{x}, a \in A$, which implies (by the ergodicity of $A \curvearrowright G / \Gamma$ ) that $c_{0} \in C$ is constant. Therefore, $\phi: G / \Gamma \rightarrow G / A$ is a constant map as well. Translating back to $f$ one obtains that $f: G / A \rightarrow G / A$ is a $G$-map, and therefore is given by $f(g A)=g n A$ for some $n \in \mathcal{N}_{G}(A)$. Thus we obtain a surjective homomorphism $\mathcal{N}_{G}(A) \rightarrow \operatorname{Map}_{\Gamma}(G / A, G / A)$ with $A$ being the kernel. This completes the proof of Theorem 1.9.

\section{Examples}

In this section we give several elementary examples which illustrate that the assumptions imposed in our main results are essential.

\section{Example 8.1}

This example demonstrates that a fat complement $M$ may be of infinite index, and that the subgroup $M$ in Theorem 1.7 may in general be of infinite index. Consider an $S$-algebraic group $\mathbf{G}=\mathbf{H} \times \mathbf{L}$ such that $H$ is compact, set $\Gamma=L$, and set $Y=G$. Then

$$
\Phi: G / H \rightarrow Y, \quad l H \mapsto(e, l), \quad l \in L,
$$

defines a $\Gamma$-map. The largest group which could play the role of $M$ in the statement is $M=L$.

\section{Example 8.2}

This example shows that the group $M$ in Theorem 1.6 does not have to be f.i.algebraic. Let $\mathbf{G}=\mathrm{SO}_{n} \times \mathrm{G}_{a}$ where $\mathrm{SO}_{n}$ denotes the orthogonal group and $\mathrm{G}_{a}$ denotes the additive group. We fix a compact one-parameter subgroup $\{k(t)\}_{t \in \mathbb{R}}$ of $\mathrm{SO}_{n}(\mathbb{R})$ and set $\Gamma=\{(k(t), t)\}_{t \in \mathbb{R}}$. We also set $H=\mathrm{SO}_{n}$. Then the map

$$
\Phi: G / H \rightarrow G, \quad(e, t) H \mapsto(k(t), t), \quad t \in \mathbb{R},
$$


is $\Gamma$-equivariant, but not equivariant almost everywhere with respect to any larger subgroup.

\section{Example 8.3}

This example shows that, even when $G$ in Theorem 1.6 has no nontrivial compact $S$-algebraic quotients, the subgroup $M$ could be proper (cf. Theorem 6.4). Let $G=$ $\mathrm{G}_{m}$, let $H=\langle \pm 1\rangle$, let $\Gamma=\mathbb{R}_{+}^{\times}$, and let

$$
\Phi: \mathbb{R}^{\times} /\langle \pm 1\rangle \rightarrow \mathbb{R}^{\times}, \quad x\langle \pm 1\rangle \mapsto x, \quad x \in \mathbb{R}_{+}^{\times},
$$

be the factor map. Then $\Phi$ is $\mathbb{R}_{+}^{\times}$-equivariant but not $\mathbb{R}^{\times}$-equivariant.

\section{Example 8.4}

This example shows that the assumption in Corollary 1.8 that the factor is p.m.p. is essential. Let $\mathbf{G}=\mathrm{PSL}_{2}$, let $k=\mathbb{R}$, and let $H$ be the diagonal subgroup of $G$. Let $\Gamma$ be a cocompact lattice in $G$, and let $X=G / H$ be equipped with the Haar measure class $\mu$. Then $\Gamma$ acts ergodically on $X$, and Theorem 1.6 implies that every measure-preserving $\Gamma$-factor of $X$ is of the form $G / Q$ where $Q$ is a closed subgroup of $G$ such that $H$ is a normal cocompact subgroup of $Q$. Hence, there are only two measure-preserving $\Gamma$-factors of $X$ corresponding to $Q=H$ and $Q=\mathcal{N}_{G}(H)$.

On the other hand, there exists an infinite normal subgroup $\Lambda$ of $\Gamma$ such that $\Lambda$ does not act ergodically on $X$. For instance, one can take $\Lambda$ such that $\Gamma / \Lambda \simeq \mathbb{Z}^{k}$ with $k \geq 3$ (see [25]). Then there is a nontrivial measure class-preserving $\Gamma$-factor $Y=\left(X / / \Lambda, P_{\Lambda}(\mu)\right)$ (see Appendix). Since $\Lambda$ acts trivially on $Y$ and $G$ is simple, the space $Y$ cannot be isomorphic to a $G$-space.

\section{Appendix. The space of ergodic components}

Let $G$ be an lcsc group. Given a $G$-space $(X, \mu)$, we denote by $\mathcal{B}(X)$ the Boolean $\sigma$-algebra consisting of measurable sets modulo the ideal of null sets. Note that every measure class-preserving $G$-map $\Phi:(X, \mu) \rightarrow(Y, v)$ between $G$-spaces induces a $G$-equivariant Boolean- $\sigma$-algebra homomorphism $\Phi^{*}: \mathcal{B}(Y) \rightarrow \mathscr{B}(X)$ defined by $\Phi^{*}([A])=\left[\Phi^{-1}(A)\right]$ for a Borel subset $A \subset X$.

For a $G$-space $(X, \mu)$, we introduce the space of ergodic components $X / / G$. This space can be characterized by the universal property-Proposition A.6(a) below.

PROPOSITION A.5 (Ergodic decomposition)

Given a $G$-space $(X, \mu)$, there exist a standard Borel space $X / / G$ and a Borel map $P_{G}: X \rightarrow X / / G$ such that

- $\quad P_{G}(g x)=P_{G}(x)$ for all $g \in G$ and almost every $x \in X$, and

- $\quad P_{G}^{*}(\mathscr{B}(X / / G))$ is the algebra of $G$-invariant elements in $\mathcal{B}(X)$. 
Proposition A.5 is a part of folklore in ergodic theory (see, e.g., [9, Theorem 5.2] for a proof).

We shall use the following functorial properties of the space $X / / G$.

\section{PROPOSITION A.6}

(a) Let $(X, \mu)$ be a $G$-space, let $Y$ be a standard Borel space, and let $\Phi: X \rightarrow Y$ be a Borel map such that $\Phi(g x)=\Phi(x)$ for all $g \in G$ and almost every $x \in$ $X$. Then there exists a Borel map $\bar{\Phi}: X / / G \rightarrow Y$ such that $\Phi=\bar{\Phi} \circ P_{G}$ on a conull set.

(b) If $H$ is a closed normal subgroup of an lcsc group $G$ and $(X, \mu)$ is a $G$-space, then $X / / H$ is equipped with a structure of a $G$-space so that $P_{H}: X \rightarrow X / / H$ is a $G$-map.

\section{Proof}

In the proof we shall use the well-known correspondence between standard Borel spaces and Boolean algebras (see [12], [19]). Specifically, we shall use the following.

(1) If $\Phi, \Psi:(X, \mu) \rightarrow(Y, v)$ are $G$-maps such that $\Phi^{*}=\Psi^{*}$, then $\Phi=\Psi$ on a conull set (see [19, Theorem 2.1]).

(2) If $(X, \mu)$ and $(Y, v)$ are $G$-spaces and $\phi: \mathscr{B}(Y) \rightarrow \mathscr{B}(X)$ is a $G$-equivariant homomorphism, then there exists a measure class-preserving $G$-map such that $\Phi^{*}=\phi($ see [12, Theorem 3], [19, Theorem 3.6]).

(3) Every Boolean space equipped with a $G$-action is $G$-equivariantly isomorphic to a Boolean measure space associated with a $G$-space (see [12, Theorem 1], [19, Theorem 3.3]).

Now we start with the proof of (a). By [33, Proposition B.5] there exists a measurable map $\Psi: X \rightarrow Y$ such that $\Psi=\Phi$ on a conull set and $\Psi$ is $G$-equivariant on a $G$-invariant Borel set of full measure. This implies that the Boolean $\sigma$-algebra $\Phi^{*}(\mathscr{B}(Y))$ consists of $G$-invariant elements; that is,

$$
\Phi^{*}(\mathscr{B}(Y)) \subset P_{G}^{*}(\mathscr{B}(X / / G))
$$

Let $\bar{\phi}: \mathscr{B}(Y) \rightarrow \mathscr{B}(X / / G)$ be the corresponding embedding. By (2) there exists a $G$-map $\bar{\Phi}: X / / G \rightarrow Y$ such that $\bar{\Phi}^{*}=\phi$. Then $P_{G}^{*} \circ \bar{\Phi}^{*}=\left(\bar{\Phi} \circ P_{G}\right)^{*}=\Phi^{*}$, and it follows from (1) that $\bar{\Phi} \circ P_{G}=\Phi$ on a conull set, as required.

Let $H$ be a closed normal subgroup of $G$. Since $P_{H}^{*}(\mathscr{B}(X / / H))$ is the algebra of $H$-invariant elements, it is invariant under $G$. This defines an action of $G$ on $\mathscr{B}(X / / H)$ such that the map $P_{H}^{*}: \mathscr{B}(X / / H) \rightarrow \mathscr{B}(X)$ is $G$-equivariant. By (3), the $G$-action on $\mathcal{B}(X / / H)$ comes from a structure of a $G$-space on $X / / H$. Since, for every $g \in G$, we have that $g^{*} \circ P_{H}^{*}=P_{H}^{*} \circ g^{*}$, it follows from (1) that $P_{G} \circ g=$ $g \circ P_{G}$ on a conull set. This completes the proof. 
COROLLARY A. 7

Let $G$ be an lcsc group, let $H$ be its closed subgroup, and let $\bar{H}^{\mathrm{MT}}$ be the Mautner envelope of $H$ as in Section 5. Let $(X, \xi)$ be a $G$-space with a $G$-invariant probability measure. Then the natural map $X / / H \rightarrow X / / \bar{H}^{\mathrm{MT}}$ is a Borel isomorphism.

\section{Proof}

To prove the corollary, we need to show that, for every Borel subset $B$ of $X$ such that $\mu(B \triangle h B)=0$ for $h \in H$, we have that $\mu(B \triangle h B)=0$ for $h \in \bar{H}^{\mathrm{MT}}$. Indeed, the characteristic function of $B$ is an $H$-invariant vector in $L^{2}(X, \mu)$; hence it is also invariant by $\bar{H}^{\mathrm{MT}}$.

Proof

Since $N<\bar{H}^{\mathrm{MT}}$, the natural map $X / / \bar{H}^{\mathrm{MT}} \rightarrow(X / / N) / / \bar{H}^{\mathrm{MT}}$ is an isomorphism. Since, by Corollary A.7, $X / / H \simeq X / / \bar{H}^{\mathrm{MT}}$ and $(X / / N) / / H \simeq(X / / N) / / \bar{H}^{\mathrm{MT}}$, this implies the claim.

Acknowledgments. We would like to thank the organizers of the meeting Rigidity, dynamics, and groups actions in July 2005 at Banff International Research Station (BIRS), during which this project was conceived. Most of the research described in this paper was conducted at Banff in June 2006 under the "research in teams" program. We would like to thank BIRS for a great time and superb working conditions.

This research was supported by Israel Science Foundation grants 584/04 and 704/08, Binational Science Foundation grant 2008267, National Science Foundation grants DMS 0905977 and 1207803, Engineering and Physical Sciences Research Council grant EP/H000091/1, and European Research Council grant 239606.

\section{References}

[1] I. AGOL, The virtual Haken conjecture, with an appendix by I. Agol, D. Groves, and J. Manning, Doc. Math. 18 (2013), 1045-1087. MR 3104553. (124)

[2] U. BADER and Y. SHALOM, Factor and normal subgroup theorems for lattices in products of groups, Invent. Math. 163 (2006), 415-454. MR 2207022. DOI 10.1007/s00222-005-0469-5. (122)

[3] Y. BENOIST and J.-F. QUINT, Mesures stationnaires et fermés invariants des espaces homogènes, II, C. R. Math. Acad. Sci. Paris 349 (2011), 341-345. MR 2783332. DOI 10.1016/j.crma.2011.01.015. (148)

[4] A. BOREL, Density properties for certain subgroups of semi-simple groups without compact components, Ann. of Math. (2) 72 (1960), 179-188. MR 0123639. (122)

[5] - Linear Algebraic Groups, 2nd ed., Grad. Texts in Math. 126, Springer, New York, 1991. MR 1102012. DOI 10.1007/978-1-4612-0941-6. (140, 141, 144) 
[6] S. G. DANI, A simple proof of Borel's density theorem, Math. Z. 174 (1980), 81-94. MR 0591617. DOI 10.1007/BF01215084. (122)

[7] A. FURMAN, Measurable rigidity of actions on infinite measure homogeneous spaces, II, J. Amer. Math. Soc. 21 (2008), 479-512. MR 2373357.

DOI 10.1090/S0894-0347-07-00588-7. (115, 122, 123)

[8] H. FURSTENBerg, A note on Borel's density theorem, Proc. Amer. Math. Soc. 55 (1976), 209-212. MR 0422497. (122)

[9] G. GRESCHONIG and K. SCHMIDT, Ergodic decomposition of quasi-invariant probability measures, Colloq. Math. 84/85 (2000), 495-514. MR 1784210. (152)

[10] Y. GUIVARC'H, Propriétés ergodiques, en mesure infinie, de certains systèmes dynamiques fibrés, Ergodic Theory Dynam. Systems 9 (1989), 433-453. MR 1016662. DOI 10.1017/S0143385700005083. (124)

[11] A. LUBOTZKY, Eigenvalues of the Laplacian, the first Betti number and the congruence subgroup problem, Ann. of Math. (2) 144 (1996), 441-452. MR 1418904. DOI 10.2307/2118597. (124)

[12] G. W. MACKEY, Point realizations of transformation groups, Illinois J. Math. 6 (1962), 327-335. MR 0143874. (152)

[13] G. A. MARGULIS, Finiteness of quotient groups of discrete subgroups, Funktsional. Anal. i Prilozhen 13 (1979), 28-39. MR 0545365. (122)

[14] - Discrete Subgroups of Semisimple Lie Groups, Ergeb. Math. Grenzgeb. (3) 17, Springer, Berlin, 1991. MR 1090825. DOI 10.1007/978-3-642-51445-6. (141)

[15] G. A. MARGULIS and G. M. TOMANOV, Measure rigidity for almost linear groups and its applications, J. Anal. Math. 69 (1996), 25-54. MR 1428093.

DOI 10.1007/BF02787100. (136, 137)

[16] A. NEVO and R. J. ZIMMER, A generalization of the intermediate factors theorem, J. Anal. Math. 86 (2002), 93-104. MR 1894478. DOI 10.1007/BF02786645. (122)

[17] R. R. PHELPS, Lectures on Choquet's Theorem, 2nd ed., Lecture Notes in Math. 1757, Springer, Berlin, 2001. MR 1835574. DOI 10.1007/b76887. (131)

[18] V. PLATONOV and A. RAPINCHUK, Algebraic Groups and Number Theory, Pure Appl. Math. (Amst.) 139, Academic, Boston, 1994. MR 1278263. (135, 139)

[19] A. RAMSAY, Virtual groups and group actions, Adv. Math. 6 (1971), 253-322. MR 0281876. (152)

[20] M. RATNER, Factors of horocycle flows, Ergodic Theory Dynam. Systems 2 (1982), 465- 489. MR 0721735. DOI 10.1017/S0143385700001723. (121)

[21] - Rigidity of horocycle flows, Ann. of Math. (2) 115 (1982), 597-614. MR 0657240. DOI 10.2307/2007014. (121, 138)

[22] - Horocycle flows, joinings and rigidity of products, Ann. of Math. (2) 118 (1983), 277-313. MR 0717825. DOI 10.2307/2007030. (121)

[23] _ "Interactions between ergodic theory, Lie groups, and number theory" in Proceedings of the International Congress of Mathematicians, Vol. 2 (Zürich, 1994), Birkhäuser, Basel, 1995, 157-182. MR 1403920. (121, 146)

[24] Raghunathan's conjectures for Cartesian products of real and p-adic Lie groups, Duke Math. J. 77 (1995), 275-382. MR 1321062.

DOI 10.1215/S0012-7094-95-07710-2. (144) 
[25] M. REES, Checking ergodicity of some geodesic flows with infinite Gibbs measure, Ergodic Theory Dynam. Systems 1 (1981), 107-133. MR 0627791. (124, 151)

[26] N. A. SHAH, "Invariant measures and orbit closures on homogeneous spaces for actions of subgroups generated by unipotent elements" in Lie Groups and Ergodic Theory (Mumbai, 1996), Tata Inst. Fund. Res. Stud. Math. 14, Tata Inst. Fund. Res., Bombay, 1998, 229-271. MR 1699367. (146)

[27] Y. SHALOM, Invariant measures for algebraic actions, Zariski dense subgroups and Kazhdan's property (T), Trans. Amer. Math. Soc. 351 (1999), no. 8, 3387-3412. MR 1615966. DOI 10.1090/S0002-9947-99-02363-6. (122, 126, 131, 132)

[28] Y. SHALOM and T. STEGER, Measurable rigidity of actions on infinite measure homogeneous spaces, I, unpublished. $(115,122)$

[29] S. P. WANG, On density properties of $S$-subgroups of locally compact groups, Ann. of Math. (2) 94 (1971), 325-329. MR 0291351. (122, 137, 138)

[30] - On the Mautner phenomenon and groups with property (T), Amer. J. Math. 104 (1982), 1191-1210. MR 0681733. DOI 10.2307/2374057. (136)

[31] D. WITTE, Measurable quotients of unipotent translations on homogeneous spaces, Trans. Amer. Math. Soc. 345 (1994), no. 2, 577-594. MR 1181187. DOI 10.2307/2154988. (121, 146)

[32] R. J. ZIMMER, Ergodic theory, semisimple Lie groups, and foliations by manifolds of negative curvature, Inst. Hautes Études Sci. Publ. Math. 55 (1982), 37-62. MR 0672181. (122)

[33] _ Ergodic Theory and Semisimple Groups, Monogr. Math. 81, Birkhäuser, Basel, 1984. MR 0776417. DOI 10.1007/978-1-4684-9488-4. (116, 127, 128, 152)

Bader

Technion, Haifa, Israel 32000; bader@tx.technion.ac.il

Furman

University of Illinois at Chicago, Chicago, Illinois 60607-7045, USA; furman@math.uic.edu

Gorodnik

University of Bristol, Bristol BS8 1TW, United Kingdom; a.gorodnik@bristol.ac.uk

Weiss

Ben Gurion University, Be’er Sheva, Israel 84105; barakw@math.bgu.ac.il 
